\title{
Rubber friction: role of the flash temperature
}

\author{
B N J Persson \\ IFF, FZ-Jülich, 52425 Jülich, Germany \\ Received 18 May 2006, in final form 7 July 2006 \\ Published 31 July 2006 \\ Online at stacks.iop.org/JPhysCM/18/7789
}

\begin{abstract}
When a rubber block is sliding on a hard rough substrate, the substrate asperities will exert time-dependent deformations of the rubber surface resulting in viscoelastic energy dissipation in the rubber, which gives a contribution to the sliding friction. Most surfaces of solids have roughness on many different length scales, and when calculating the friction force it is necessary to include the viscoelastic deformations on all length scales. The energy dissipation will result in local heating of the rubber. Since the viscoelastic properties of rubber-like materials are extremely strongly temperature dependent, it is necessary to include the local temperature increase in the analysis. At very low sliding velocity the temperature increase is negligible because of heat diffusion, but already for velocities of order $10^{-2} \mathrm{~m} \mathrm{~s}^{-1}$ the local heating may be very important. Here I study the influence of the local heating on the rubber friction, and I show that in a typical case the temperature increase results in a decrease in rubber friction with increasing sliding velocity for $v>0.01 \mathrm{~m} \mathrm{~s}^{-1}$. This may result in stick-slip instabilities, and is of crucial importance in many practical applications, e.g. for tyre-road friction and in particular for ABS braking systems.
\end{abstract}

\section{Introduction}

Rubber friction is of extreme practical importance, e.g. in the context of tyres, wiper blades, conveyor belts and seals [1]. Rubber friction on smooth substrates, e.g., on smooth glass surfaces, has two contributions, namely an adhesive (surface) and a hysteresis (bulk) contribution [1,2]. The adhesive contribution results from the attractive binding forces between the rubber surface and the substrate. Surface forces are often dominated by weak attractive van der Waals interactions. For very smooth substrates, because of the low elastic moduli of rubberlike materials, even when the applied squeezing force is very gentle this weak attraction may result in a nearly complete contact at the interface [3-5], leading to the large sliding friction force usually observed [6]. For rough surfaces, on the other hand, the adhesive contribution 
to rubber friction will be much smaller because of the small contact area ${ }^{1}$. The actual contact area between a tyre and the road surface, for example, is typically only $\sim 1 \%$ of the nominal footprint contact area [7-10]. Under these conditions the bulk (hysteresis) friction mechanism is believed to prevail $[8,10]$ (see footnote 1 ). For example, the exquisite sensitivity of tyre-road friction to temperature just reflects the strong temperature dependence of the viscoelastic bulk properties of rubber.

When a rubber block is slid on a hard rough substrate the surface asperities of the substrate will exert fluctuating forces on the rubber surface which, because of the internal friction of the rubber, will result in energy transfer from the translational motion of the block into the irregular thermal motion. This will result in a contribution to the friction force acting on the rubber block. The energy dissipation will result in local heating of the rubber. Since the viscoelastic properties of rubber-like materials are extremely strongly temperature dependent, it is necessary to include the local temperature increase when calculating the friction force. At very low sliding velocity the temperature increase is negligible because of heat diffusion, but already for velocities of order $10^{-2} \mathrm{~m} \mathrm{~s}^{-1}$ the local heating may become very important. Here I study the influence of the local heating on the rubber friction. I show that in a typical case the temperature increase will result in a friction which decreases with increasing sliding velocity for $v>0.01 \mathrm{~m} \mathrm{~s}^{-1}$. This may result in stick-slip instabilities, and is of crucial importance in many practical applications, e.g. for tyre-road friction, and in particular for ABS braking systems.

\section{Surface roughness and macro-asperity contact}

The influence of surface roughness on rubber friction is mainly determined by the surface roughness power spectrum $C(q)$ defined by

$$
C(q)=\frac{1}{(2 \pi)^{2}} \int \mathrm{d}^{2} x\langle h(\mathbf{x}) h(\mathbf{0})\rangle \mathrm{e}^{-\mathrm{i} \mathbf{q} \cdot \mathbf{x}} .
$$

Here $h(\mathbf{x})$ is the substrate height measured from the average plane defined so that $\langle h\rangle=0$. The $\langle\cdots\rangle$ stand for ensemble averaging, or averaging over the surface area. We have assumed that the statistical properties of the substrate are translationally invariant and isotropic so that $C(q)$ only depend on the magnitude $q=|\mathbf{q}|$ of the wavevector $\mathbf{q}$. Note that from (1) it follows that

$$
\langle h(\mathbf{x}) h(\mathbf{0})\rangle=\int \mathrm{d}^{2} q C(q) \mathrm{e}^{\mathrm{i} \mathbf{q} \cdot \mathbf{x}}
$$

so that the root-mean-square (rms) roughness amplitude $h_{0}=\left\langle h^{2}\right\rangle^{1 / 2}$ is determined by

$$
\left\langle h^{2}\right\rangle=\int \mathrm{d}^{2} q C(q)=2 \pi \int_{0}^{\infty} \mathrm{d} q q C(q) .
$$

In reality, there will always be an upper, $q_{1}$, and a lower, $q_{L}$, limit to the $q$-integral in (2). Thus, the largest possible wavevector will be of order $2 \pi / a$, where $a$ is some lattice constant, and the smallest possible wavevector is of order $2 \pi / L$, where $L$ is the linear size of the surface.

Many surfaces tend to be nearly self-affine fractal. A self-affine fractal surface has the property that if part of the surface is magnified, with a magnification which in general is appropriately different in the perpendicular direction to the surface as compared to the lateral directions, then the surface 'looks the same', i.e. the statistical properties of the surface are

1 The role of the adhesional contribution to rubber friction is still controversial. Thus in [9] it is argued that the opening cracks in the contact regions between the substrate (e.g road) asperities and the sliding rubber block may strongly enhance the contribution from the adhesional interaction to the sliding friction. 


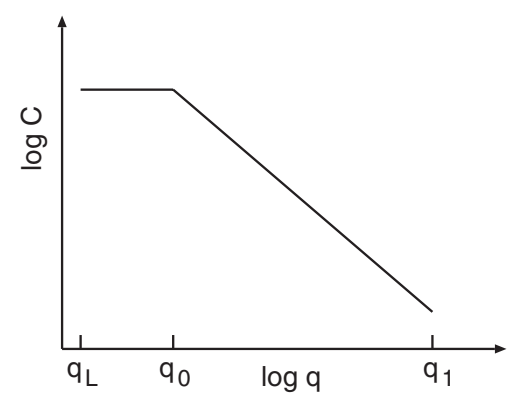

Figure 1. Surface roughness power spectrum of a surface which is self-affine fractal for $q_{1}>q>$ $q_{0}$. The long distance roll-off wavevector $q_{0}$ and the short distance cut-off wavevector $q_{1}$ depend on the system under consideration. The slope of the $\log C-\log q$ relation for $q>q_{0}$ determines the fractal exponent of the surface. The lateral size $L$ of the surface (or of the studied surface region) determines the smallest possible wavevector $q_{L}=2 \pi / L$.

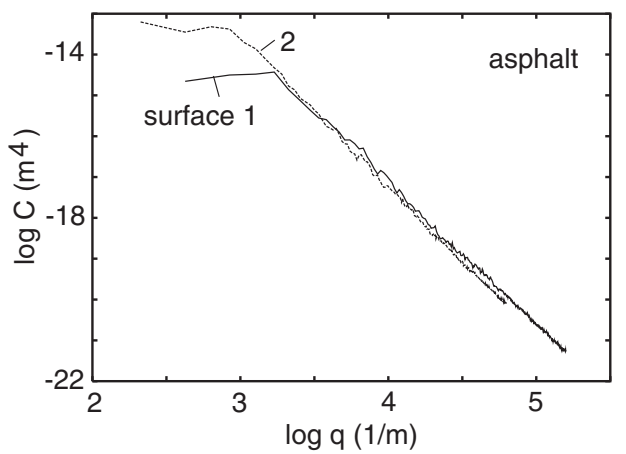

Figure 2. The surface roughness power spectra $C(q)$ for two asphalt road surfaces.

invariant under the scale transformation. For a self-affine surface the power spectrum has the power-law behaviour

$$
C(q) \sim q^{-2(H+1)},
$$

where the Hurst exponent $H$ is related to the fractal dimension $D_{\mathrm{f}}$ of the surface via $H=$ $3-D_{\mathrm{f}}$. Of course, for real surfaces this relation only holds in some finite wavevector region $q_{0}<q<q_{1}$, and in a typical case $C(q)$ has the form shown in figure 1. Note that in many cases there is a roll-off wavevector $q_{0}$ below which $C(q)$ is approximately constant.

Asphalt and concrete road pavements have nearly perfect self-affine fractal power spectra, with very well defined roll-off wavevector $q_{0}=2 \pi / \lambda_{0}$ of order $1000 \mathrm{~m}^{-1}$, corresponding to $\lambda_{0} \approx 1 \mathrm{~cm}$, which reflect the largest stone particles used in the asphalt. This is illustrated in figure 2 for two different asphalt pavements. From the slope of the curves for $q>q_{0}$ one can deduce the fractal dimension $D_{\mathrm{f}} \approx 2.2$, which is typical for asphalt and concrete road surfaces.

Assume that an elastic solid with a flat surface is squeezed against a hard, randomly rough substrate. Figure 3 shows the contact between two solids at increasing magnification $\zeta$. At low magnification $(\zeta \approx 1)$ it looks as if complete contact occurs between the solids at many macroasperity contact regions, but when the magnification is increased smaller length scale roughness is detected, and it is observed that only partial contact occurs at the asperities. In fact, if there were no short distance cut-off the true contact area would vanish. In reality, however, a short distance cut-off will always exist since the shortest possible length is an atomic distance. For 


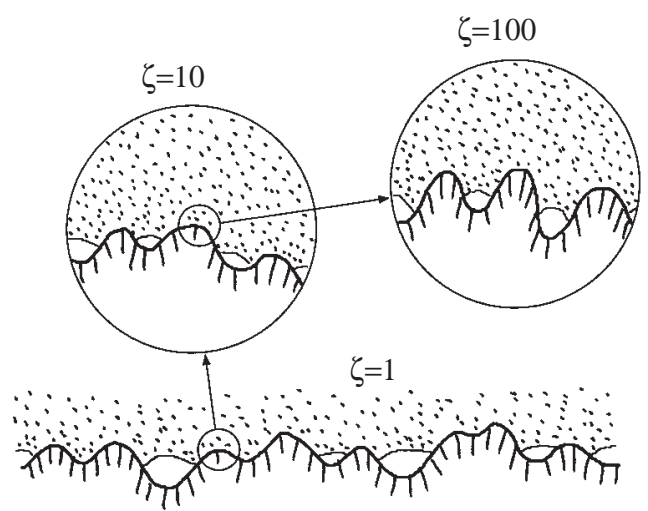

Figure 3. A rubber block (dotted area) in adhesive contact with a hard rough substrate (dashed area). The substrate has roughness on many different length scales and the rubber makes partial contact with the substrate on all length scales. When a contact area is studied at low magnification $(\zeta=1)$ it appears as if complete contact occurs in the macro-asperity contact regions, but when the magnification is increased it is observed that in reality only partial contact occurs.

rubber friction the effective short distance cut-off may be much larger (of micrometre order); in section 10 we will argue that it is related to the modification (degradation) of a thin rubber surface layer due to the high stresses and temperatures (and environmental gases) it is exposed to during sliding.

The magnification $\zeta$ refers to some (arbitrarily) chosen reference length scale. This could be, e.g., the lateral size $L$ of the nominal contact area in which case $\zeta=L / \lambda$, where $\lambda$ is the shortest roughness wavelength component which can be resolved at magnification $\zeta$. In this paper we will consider surfaces with power spectra of the form shown in figure 1 , and we will use the roll-off wavelength $\lambda_{0}=2 \pi / q_{0}$ as the reference length so that $\zeta=\lambda_{0} / \lambda=q / q_{0}$.

I now explain the concept of macro-asperity contact area, which is important for my treatment of the influence of the flash temperature on rubber friction. Assume that the surface roughness has the qualitative form shown in figure 1 with a roll-off wavevector $q_{0}$ corresponding to the magnification $\zeta=1$. In this case the macro-asperity contact is the contact region between the solids when the system is studied at low magnification $\zeta=\zeta_{\mathrm{m}} \approx 2$ 5 (see appendix A). At this magnification, at low squeezing pressures one observes a dilute distribution of randomly distributed macro-asperity contact regions with lateral size typically of order $\sim \lambda_{0} / \zeta_{\mathrm{m}}$. As the magnification is increased each macro-asperity contact region breaks up into a relative dense distribution of much smaller micro-asperity contact regions. This is illustrated in figure 4 which shows the result of a molecular dynamics (MD) calculation [11] where the surface roughness power spectrum was assumed to be of the form shown in figure 1 (with the fractal dimension 2.2).

Consider the contact between two solids at low nominal contact pressure $\sigma_{0}=F_{\mathrm{N}} / A_{0}$, where the contact area is proportional to the load. Consider first the system on the length scale $\lambda_{0} / \zeta_{\mathrm{m}}$. On this length scale the solids will make (apparent) contact at a low concentration of (widely separated) contact areas. Since the separation between these macro-asperity contact regions is very large we can neglect the interaction between the macro-contact regions: in this case the pressure in the macro-asperity contact regions will be of order $\sim q_{0} h_{0} E$, where $h_{0}$ is the rms roughness amplitude, and $E$ the elastic modulus. Thus, the (average) pressure in the macro-asperity contact regions is independent of the nominal contact pressure $\sigma_{0}=F_{\mathrm{N}} / A_{0}$. Now, each macro-asperity is covered by smaller micro-asperities, and the smaller asperities 


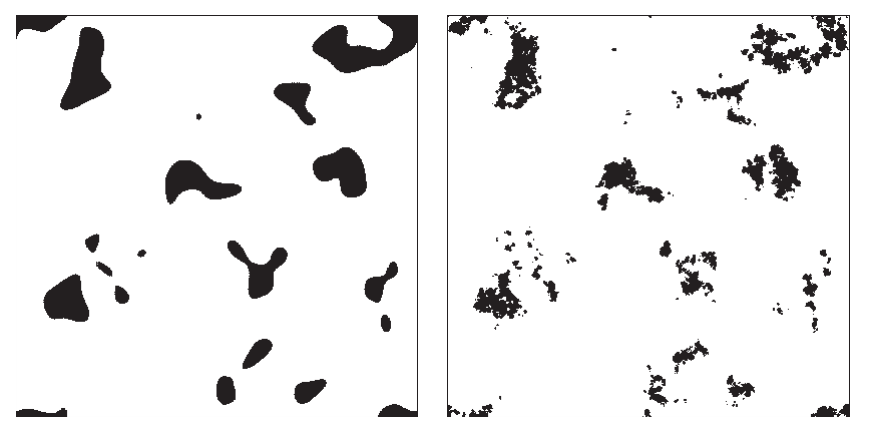

Figure 4. The contact area between an elastic solid with a flat surface and a hard randomly rough substrate shown at low magnifications $(\zeta=4)$, left, and high magnification $(\zeta=216)$, right. The surface has the fractal dimension $D_{\mathrm{f}}=2.2$ and $q_{0} / q_{L}=3$. Adapted from [11].

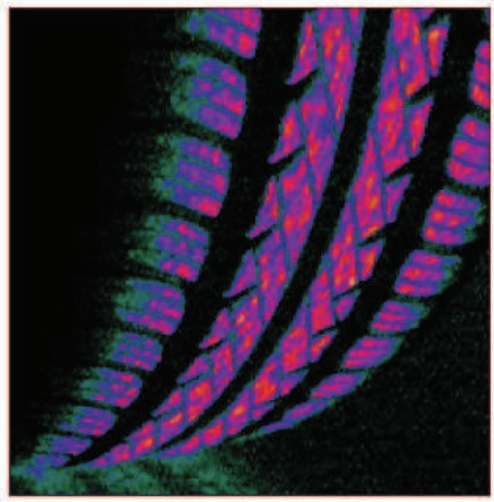

Figure 5. Infrared photograph of tyre as it rotates out of contact with the road. The red-yellow colour indicates the 'hot' spots arising from macro-asperity contacts in the tyre-road footprint. Adapted from $[12,13]$.

by even smaller asperities, and so on. It is easy to see that the micro-asperity contact regions will be very closely separated and it is therefore impossible to neglect the (elastic or thermal) interaction between the asperities at short enough length scale.

In section 4 we will calculate the flash temperature in the asperity contact regions as a rubber block is sliding on a rough substrate. We will neglect the thermal coupling (or overlap) between the macro-asperity contact regions, which should be a good approximation as long as the contact area at the magnification $\zeta \approx \zeta_{\mathrm{m}}$ is much smaller than the nominal contact area (which implies that the distance between the macro-asperity contact regions is much larger than the linear size $\sim \lambda_{0} / \zeta_{\mathrm{m}}$ of these regions). However, owing to the high density of micro-asperity contact regions within a macro-asperity contact region, it is not possible to neglect the thermal coupling (or overlap) between the micro-asperity contact regions within each macro-asperity asperity contact region.

The temperature increase in the macro-asperity contact regions between a tyre and a road surface has recently been studied using an infrared camera. Figure 5 shows a photograph of a tyre as it rotates out of contact with the road. The red-yellow colour indicate the 'hot' spots arising from macro-asperity contacts in the tyre-road footprint. 


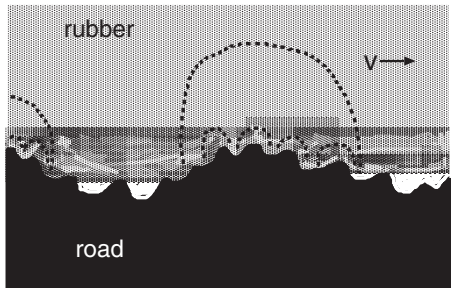

Figure 6. The road asperities exert pulsating forces on the sliding rubber block, leading to energy dissipation in the rubber via the internal friction of the rubber. Most of the energy dissipation occurs in the volume elements bound by the dashed lines. The rubber viscoelastic deformations in the large volume elements are induced by the large road asperities, while the smaller dissipative regions result from the smaller asperities distributed on top of the large asperities. In general, in calculating the rubber friction, the viscoelastic energy dissipation induced by all the asperity sizes must be included, and the local temperature increase (flash temperature) in the rubber resulting from the energy dissipation must also be taken into account in the analysis.

\section{Rubber friction without flash temperature}

The main contribution to rubber friction when a rubber block is sliding on a rough substrate, i.e. a tyre on a road surface, is due to the viscoelastic energy dissipation in the surface region of the rubber as a result of the pulsating forces acting on the rubber surface from the substrate asperities, see figure 6. Recently I have developed a theory which accurately describes this energy dissipation process, and which predicts the velocity dependence of the rubber friction coefficient $[7,10]$. The results depend only on the (complex) viscoelastic modulus $E(\omega)$ of the rubber, and on the substrate surface roughness power spectrum $C(q)$. Neglecting the flashtemperature effect, the kinetic friction coefficient at velocity $v$ is determined by [10]

$$
\mu_{\mathrm{k}}=\frac{1}{2} \int_{q_{L}}^{q_{1}} \mathrm{~d} q q^{3} C(q) P(q) \int_{0}^{2 \pi} \mathrm{d} \phi \cos \phi \operatorname{Im} \frac{E(q v \cos \phi)}{\left(1-v^{2}\right) \sigma_{0}},
$$

where

$$
P(q)=\frac{2}{\pi} \int_{0}^{\infty} \mathrm{d} x \frac{\sin x}{x} \exp \left(-x^{2} G\right)=\operatorname{erf}\left(\frac{1}{2 \sqrt{ } G}\right)
$$

with

$$
G(q)=\frac{1}{8} \int_{q_{L}}^{q} \mathrm{~d} q q^{3} C(q) \int_{0}^{2 \pi} \mathrm{d} \phi\left|\frac{E(q v \cos \phi)}{\left(1-v^{2}\right) \sigma_{0}}\right|^{2},
$$

where $\sigma_{0}$ is the mean perpendicular pressure (load divided by the nominal contact area), and $v$ the Poisson ratio, which equals 0.5 for rubber-like materials. The factor $P(q)=P\left(\zeta q_{0}\right)=$ $A(\zeta) / A_{0}$ is the (normalized) area of contact when the system is studied at the magnification $\zeta=q / q_{0}$.

The theory takes into account the substrate surface roughness components with wavevectors in the range $q_{L}<q<q_{1}$, where $q_{L}$ is the smallest relevant wavevector of order $2 \pi / L$, where (in the case of a tyre) $L$ is the lateral size of a tread block, and where $q_{1}$ may have different origins (see below). Since $q_{L}$ for a tyre tread block is smaller than the roll-off wavevector $q_{0}$ of the power spectra of most road surfaces (see figure 2), rubber friction is very insensitive to the exact value of $q_{L}$.

The large wavevector cut-off $q_{1}$ may be related to road contamination, or may be an intrinsic property of the tyre rubber. For example, if the road surface is covered by small contamination particles (diameter $D$ ) then $q_{1} \approx 2 \pi / D$. In this case, the physical picture is 


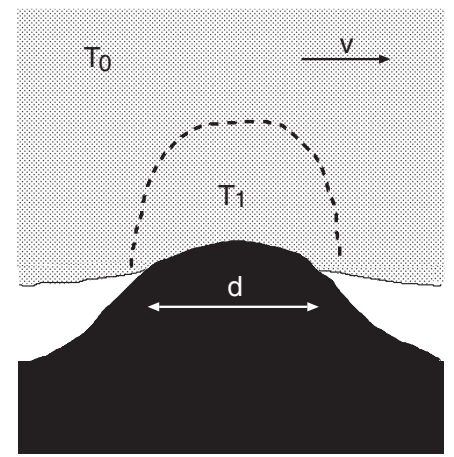

Figure 7. A rubber block sliding on a rough hard substrate with surface roughness on a single length scale. The region in the vicinity of a substrate asperity is shown. The heat energy production $\dot{Q}(\mathbf{x}, t)$ per unit volume and unit time occurs mainly within the volume element surrounded by a dashed line.

that when the tyre rubber surface is covered by hard particles of linear size $D$ the rubber will not be able to penetrate into surface roughness 'cavities' with diameter (or wavelength) smaller than $D$, and such short-range roughness will therefore not contribute to the rubber friction. For perfectly clean road surfaces we believe instead that the cut-off $q_{1}$ is related to the tyre rubber properties. Thus, the high local (flash) temperatures and high local stresses which occur in the tyre rubber-road asperity contact regions (see below) may result in a thin (typically of order a few micrometres) surface layer of rubber with modified properties (a 'dead' layer), which may contribute very little to the observed rubber friction (see section 10). Since the stresses and temperatures which develop in the asperity contact regions depend somewhat on the type of road (via the surface roughness power spectrum $C(q)$ ), the thickness of this 'dead' layer may vary from one road surface to another, and some run-in time period will be necessary for a new 'dead' layer to form when a car switches from one road surface to another (such 'run-in' effects are well known experimentally).

\section{Rubber friction with flash temperature}

The temperature field $T(\mathbf{x}, t)$ in the rubber block is determined by

$$
\frac{\partial T}{\partial t}-D \nabla^{2} T=\frac{\dot{Q}(\mathbf{x}, t)}{\rho C_{V}}
$$

where $\dot{Q}$ is the energy production per unit volume and unit time as a result of the internal friction in the rubber. The heat diffusivity $D=\lambda / \rho C_{V}$, where $\rho$ is the mass density and $\lambda$ the heat conductivity. For rubber we typically have $\rho \approx 10^{3} \mathrm{~kg} \mathrm{~m}^{-3}, C_{V} \approx 10^{3} \mathrm{~J} \mathrm{~kg}^{-1} \mathrm{~K}^{-1}$ and $\lambda \approx 0.1 \mathrm{~W} \mathrm{~m}^{-1} \mathrm{~K}^{-1}$. This gives $D \approx 10^{-7} \mathrm{~m}^{2} \mathrm{~s}^{-1}$. Consider now a rubber block with a flat surface sliding on a rough hard substrate. Assume that there is an asperity contact area with diameter $d$; see figure 7. During sliding at velocity $v$ the asperity will generate pulsating forces on the rubber surface characterized by the frequency $\omega_{0} \sim v / d$, which will result in energy dissipation in a volume element of order $d^{3}$. If the velocity $v$ is high enough, negligible heat diffusion will take place during the time period $d / v$ and the temperature increase in the rubber in the vicinity of the asperity will be $\Delta T \approx Q / \rho C_{V}$, where $Q$ is the amount of frictional heat energy (per unit volume) in the volume element $d^{3}$. The assumption of negligible heat diffusion requires that the effective contact time $d / v$ is smaller than the diffusion time $d^{2} / D$, 
i.e. $v d>D$. Thus, for example, if $v=0.1 \mathrm{~m} \mathrm{~s}^{-1}$, heat diffusion will be unimportant if $d>D / v \approx 10^{-6} \mathrm{~m}$. However, after the substrate asperity-rubber contact is 'broken', the peak temperature will decrease, and the temperature distribution will broaden with increasing time, in a way determined by the heat diffusion equation.

In this section we will develop a theory for the influence of the flash temperature on rubber friction when the substrate has roughness on very many different length scales. We consider first an idealized case where surface roughness occurs on a single length scale, and then the more complex case of roughness on many different length scales.

We consider a surface with randomness on a single length scale $\lambda_{0}=2 \pi / q_{0}$ and with the root-mean-square roughness amplitude $h_{0}$. The surface roughness power spectrum $C(q)=$ $\left(h_{0}^{2} / 2 \pi q_{0}\right) \delta\left(q-q_{0}\right)$. The (average) radius of curvature of the asperities is $R \approx 1 /\left(q_{0}^{2} h_{0}\right)$. Figure 7 shows the contact between one substrate asperity and the rubber. The diameter of the contact area is of order $d$. We assume that no roughness occurs on smaller length scale than the size of the asperity in figure 7. The time it takes to slide the distance $d$ is $d / v$. During this time the heat energy produced in the volume element $\sim d^{3}$ at the asperity is given by $\mu F_{\mathrm{N}} d$, where $F_{\mathrm{N}}=\sigma d^{2}$ is the normal asperity load ( $\sigma$ is the average perpendicular stress in the contact area). Thus the energy production per unit volume $Q \approx \mu F_{\mathrm{N}} d / d^{3}=\mu \sigma$. Neglecting heat diffusion this will result in the temperature increase $\Delta T \approx Q / \rho C_{V} \approx \mu \sigma / \rho C_{V}$. But we have shown earlier that [2]

$$
\mu \approx \sigma \frac{\operatorname{Im} E\left(\omega_{0}, T\right)}{\left|E\left(\omega_{0}, T\right)\right|^{2}}
$$

where $\omega_{0}=v q_{0}$. From standard contact mechanics theory one expects $\sigma \approx q_{0} h_{0}\left|E\left(\omega_{0}, T\right)\right|$. Thus the friction

$$
\mu \approx q_{0} h_{0} \frac{\operatorname{Im} E\left(\omega_{0}, T\right)}{\left|E\left(\omega_{0}, T\right)\right|}
$$

and the temperature increase

or

$$
\Delta T \approx\left(q_{0} h_{0}\right)^{2} \frac{\operatorname{Im} E\left(\omega_{0}, T\right)}{\rho C_{V}}
$$

$$
T \approx T_{0}+\left(q_{0} h_{0}\right)^{2} \frac{\operatorname{Im} E\left(\omega_{0}, T\right)}{\rho C_{V}}
$$

where $T_{0}$ is the background temperature. Note that the complex elastic modulus $E(\omega, T)$ depends on the (local) temperature $T$. For 'simple' (unfilled) rubber the WilliamsLandel-Ferry equation (WLF) [14] can be used to (approximately) describe the temperature dependence of $E(\omega, T)$ :

$$
E(\omega, T)=E\left(\omega a_{T} / a_{T_{0}}, T_{0}\right)
$$

where

$$
\log _{10} a_{T} \approx-8.86 \frac{T-T_{\mathrm{g}}-50}{51.5+T-T_{\mathrm{g}}} .
$$

For any given viscoelastic modulus $E\left(\omega, T_{0}\right)$, equations (7)-(9) form a complete set of equations from which the temperature $T$ and the friction coefficient $\mu$ can be obtained by, e.g., iteration. Here I will only discuss qualitatively how the temperature increase $\Delta T$ influences the rubber friction.

In order to understand the qualitative influence of the flash temperature on rubber friction it is necessary to know the general structure of the viscoelastic modulus $E(\omega)$ of rubber-like materials. In figure 8 we show the real $E_{1}=\operatorname{Re} E$ and the imaginary part $E_{2}=\operatorname{Im} E$ of 


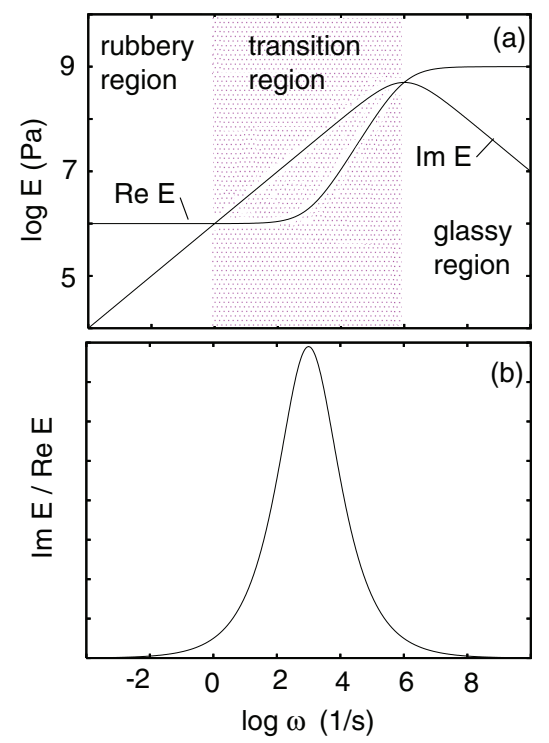

Figure 8. (a) The viscoelastic modulus $E(\omega)=E_{1}+\mathrm{i} E_{2}$ of a typical rubber-like material, and (b) the loss tangent $E_{2} / E_{1}$ (schematic).

$E(\omega)$ and also the loss tangent $E_{2} / E_{1}$. At 'low' frequencies the material is in the 'rubbery' region where $\operatorname{Re} E(\omega)$ is relatively small and approximately constant. At very high frequencies the material is elastically very stiff (brittle-like). In this 'glassy' region $\operatorname{Re} E$ is again nearly constant but much larger (typically by three to four orders of magnitude) than in the rubbery region. In the intermediate frequency range (the 'transition' region) the loss tangent is very large and it is mainly this region which determines, e.g., the friction when a tyre is sliding on a road surface.

The influence of the flash temperature on rubber friction differs depending on if the perturbing frequency $\omega_{0}=v q_{0}$ is smaller or larger than the frequency $\omega_{1}$ where $\operatorname{Im} E\left(\omega, T_{0}\right) /\left|E\left(\omega, T_{0}\right)\right|$ is maximal. Since an increase in the temperature $\left(T_{0} \rightarrow T>T_{0}\right)$ shifts the viscoelastic spectrum to higher frequencies (see the WLF function), if $\omega_{0}<\omega_{1}$ the flash-temperature will decrease the friction. On the other hand, if $\omega_{0}>\omega_{1}$ the opposite effect occur. However, in most applications, e.g. in tyre applications, the perturbing frequencies are (almost) always below $\omega_{1}$ and the friction will decrease when the flash temperature is taken into account. This has extremely important practical consequences, as will be discussed later.

We consider now the role of the flash temperature for the general (and more complex) case where roughness occurs on many different length scales. We consider first stationary sliding and then non-stationary sliding.

\subsection{Stationary sliding}

In this section we will develop a general expression for the friction acting on a rubber block sliding at a constant velocity on a randomly rough substrate. We will take into account the effect of the flash temperature. We will include the thermal overlap between the heat produced in the micro-asperity contact area inside every macro-asperity contact area. That is, the temperature rise at one micro-asperity contact area will produce a subsequent temperature rise at a neighbouring micro-contact. We will make use of a 'mean field' type of approximation 


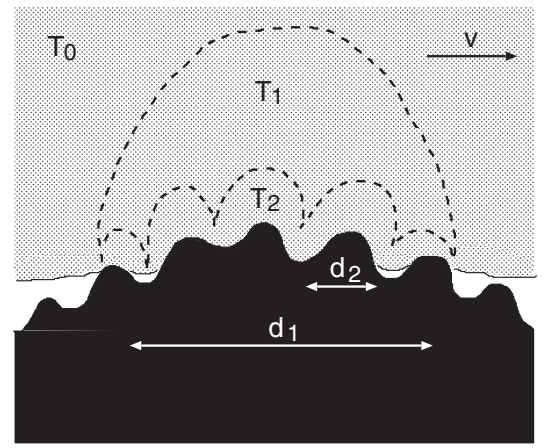

Figure 9. A rubber block sliding on an asperity with shorter wavelength asperities on top of it. The temperature increases $T_{0}<T_{1}<T_{2}$.

where we laterally smear out the heat sources associated with the micro-asperity contacts within a macro-asperity contact, which should be an excellent approximation in most cases.

Let us now discuss the flash temperature when a rubber block is sliding on a hard substrate with surface roughness on many different length scales. The basic problem is illustrated schematically in figure 9 in the case of only two length scales, where a 'large' asperity is covered by 'small' asperities. In response to the large asperity there will be a heating of the rubber in the big volume element of linear size $d_{1}$. If $T_{0}$ denotes the background temperature, then the flash temperature in the large volume element will be higher, say $T_{1}=T_{0}+\Delta T_{1}$. The flash temperature in a small asperity volume element (linear size $d_{2}$, see figure 9) will be even higher, say $T_{2}=T_{1}+\Delta T_{2}$. For surfaces with surface roughness on many length scales the temperature will increase as we go to smaller and smaller asperity contact regions. We now study the temperature distribution quantitatively.

The average shear stress which acts in the macro-asperity contact area is

$$
\sigma_{\mathrm{m}}=\sigma_{\mathrm{f}} \frac{A_{0}}{A\left(\zeta_{\mathrm{m}}\right)}
$$

where $\sigma_{\mathrm{f}}$ is the nominal frictional shear stress, and $A\left(\zeta_{\mathrm{m}}\right)$ the macro-asperity contact area observed at the magnification $\zeta=\zeta_{\mathrm{m}}=\lambda_{0} / \lambda_{\mathrm{m}}=q_{\mathrm{m}} / q_{0}$, which usually is of order unity (see appendix A). Using (3) and (10) and that $P(q)=A(\zeta) / A_{0}$ gives

$$
\sigma_{\mathrm{m}}=\frac{1}{2} \int \mathrm{d}^{2} q q^{2} \cos \phi C(q) \frac{P(q)}{P\left(q_{\mathrm{m}}\right)} \operatorname{Im} \frac{E\left(q v \cos \phi, T_{q}\right)}{1-v^{2}}
$$

where $T_{q}$ is an effective temperature in the volume of the rubber involved in the calculation of the contribution to rubber friction from surface roughness on the length scale $\lambda=2 \pi / q$ (see below). Thus the energy 'dissipation' per unit time and unit area in a macro-contact area

$$
J=\frac{v}{2} \int \mathrm{d}^{2} q q^{2} \cos \phi C(q) \frac{P(q)}{P\left(q_{\mathrm{m}}\right)} \operatorname{Im} \frac{E\left(q v \cos \phi, T_{q}\right)}{1-v^{2}} .
$$

The energy production per unit volume, from asperities on the length scale $\lambda=2 \pi / q$, decays into the solid as $\sim \mathrm{e}^{-2 q z}$, so that the energy production per unit volume and unit time in a contact area is obtained by introducing in the integrand in (12) the factor

$$
\frac{\mathrm{e}^{-2 q z}}{\int_{0}^{\infty} \mathrm{d} z \mathrm{e}^{-2 q z}}=2 q \mathrm{e}^{-2 q z} .
$$


Using this equation the energy production per unit volume and unit time in the rubber in a macro-asperity contact area is given by

$$
\dot{Q}(z, t)=\theta(t) \frac{v}{2} \int \mathrm{d}^{2} q 2 q^{3} \mathrm{e}^{-2 q z} \cos \phi C(q) \frac{P(q)}{P\left(q_{\mathrm{m}}\right)} \operatorname{Im} \frac{E\left(q v \cos \phi, T_{q}\right)}{1-v^{2}}
$$

where we have assumed that the energy production starts at $t=0$. We write the step function $\theta(t)$ as

$$
\theta(t)=-\frac{1}{2 \pi \mathrm{i}} \int \mathrm{d} \omega \frac{1}{\omega+\mathrm{i} \epsilon} \mathrm{e}^{-\mathrm{i} \omega t}
$$

where $\epsilon$ is a positive infinitesimal number. We can also write

$$
\mathrm{e}^{-2 q|z|}=\frac{1}{2 \pi} \int \mathrm{d} k \frac{4 q}{k^{2}+4 q^{2}} \mathrm{e}^{\mathrm{i} k z}
$$

Thus

$$
\frac{\dot{Q}(z, t)}{\rho C_{V}}=\int_{0}^{\infty} \mathrm{d} q f(q) \frac{(-1)}{\mathrm{i}(2 \pi)^{2}} \int \mathrm{d} \omega \mathrm{d} k \frac{1}{\omega+\mathrm{i} \epsilon} \frac{4 q}{k^{2}+4 q^{2}} \mathrm{e}^{\mathrm{i}(k z-\omega t)}
$$

where

$$
f(q)=\frac{v q^{4}}{\rho C_{V}} C(q) \frac{P(q)}{P\left(q_{\mathrm{m}}\right)} \int \mathrm{d} \phi \cos \phi \operatorname{Im} \frac{E\left(q v \cos \phi, T_{q}\right)}{1-v^{2}} .
$$

To solve the heat diffusion equation (6) we need a boundary condition on the surface $z=0$. We will assume that $\partial T / \partial z=0$ for $z=0$, i.e. we neglect heat transfer from the rubber to the substrate. This is an excellent approximation at high enough sliding velocity. At low sliding velocity it will give rise to an overestimation of the flash temperature, but in this case the flash temperature effect is anyhow not very important. The boundary condition $\partial T / \partial z(z=0)=0$ is equivalent to solving the heat diffusion equation for an extended solid $(-\infty<z<\infty)$ with a symmetric heat source obtained by replacing $\exp (-2 q z)$ in (14) by $\exp (-2 q|z|)$. Thus we get

$T(z, t)=T_{0}+\int_{0}^{\infty} \mathrm{d} q f(q) \frac{(-1)}{(2 \pi)^{2}} \int \mathrm{d} \omega \mathrm{d} k \frac{1}{\omega+\mathrm{i} D k^{2}} \frac{1}{\omega+\mathrm{i} \epsilon} \frac{4 q}{k^{2}+4 q^{2}} \mathrm{e}^{\mathrm{i}(k z-\omega t)}$.

Performing the $\omega$-integration gives

$T(z, t)=T_{0}+\int_{0}^{\infty} \mathrm{d} q f(q) \frac{1}{2 \pi} \int \mathrm{d} k \frac{1}{D k^{2}}\left(1-\mathrm{e}^{-D k^{2} t}\right) \frac{4 q}{k^{2}+4 q^{2}} \mathrm{e}^{\mathrm{i} k z}$.

The optimum (spatially uniform) temperature to be used when calculating the contribution to the friction from surface roughness on the length scale $\lambda=2 \pi / q$ can be obtained using

$$
T_{q}=\frac{\int_{0}^{\infty} \mathrm{d} z T(z, t) \mathrm{e}^{-2 q z}}{\int_{0}^{\infty} \mathrm{d} z \mathrm{e}^{-2 q z}}
$$

Using (18) this gives

$$
T_{q}=T_{0}+\int_{0}^{\infty} \mathrm{d} q^{\prime} g\left(q, q^{\prime}\right) f\left(q^{\prime}\right)
$$

where

$$
g\left(q, q^{\prime}\right)=\frac{1}{\pi} \int_{0}^{\infty} \mathrm{d} k \frac{1}{D k^{2}}\left(1-\mathrm{e}^{-D k^{2} t_{0}}\right) \frac{4 q^{\prime}}{k^{2}+4 q^{\prime 2}} \frac{4 q^{2}}{k^{2}+4 q^{2}}
$$

where $t_{0} \approx R / v$ is roughly half the time a rubber patch is in contact with the macro-asperity. The radius $R$ of a macro-asperity contact region is estimated in appendix A. Note that the complex elastic modulus $E(\omega, T)$ depends on the (local) temperature $T$. In general one may 
write $E(\omega, T)=b_{T} E\left(\omega a_{T}, T_{0}\right)$, where $a_{T}$ and $b_{T}$ depend on the temperature $T$ but with $a_{T_{0}}=b_{T_{0}}=1$. For unfilled rubber $b_{T} \approx 1$ and $a_{T}$ is (approximately) given by the WLF equation. The functions $a_{T}$ and $b_{T}$ are today measured routinely using standard rheological equipment.

When $T_{q}$ has been obtained from (20) and (21), the friction coefficient can be calculated using the equations derived in [10]:

$$
\begin{aligned}
& \mu \approx \frac{1}{2} \int_{q_{0}}^{q_{1}} \mathrm{~d} q q^{3} C(q) P(q) \int_{0}^{2 \pi} \mathrm{d} \phi \cos \phi \operatorname{Im} \frac{E\left(q v \cos \phi, T_{q}\right)}{\left(1-v^{2}\right) \sigma_{0}} \\
& P(q)=\frac{2}{\pi} \int_{0}^{\infty} \mathrm{d} x \frac{\sin x}{x} \exp \left[-x^{2} G(q)\right]
\end{aligned}
$$

where

$$
G(q)=\frac{1}{8} \int_{q_{0}}^{q} \mathrm{~d} q q^{3} C(q) \int_{0}^{2 \pi} \mathrm{d} \phi\left|\frac{E\left(q v \cos \phi, T_{q}\right)}{\left(1-v^{2}\right) \sigma_{0}}\right|^{2}
$$

\subsection{Non-stationary sliding}

Here we develop a completely general theory of non-stationary sliding. Consider again the heat diffusion equation

$$
\frac{\partial T}{\partial t}-D \nabla^{2} T=\frac{\dot{Q}(z, t)}{\rho C_{V}} \theta(R-|\mathbf{x}-\mathbf{r}(t)|)
$$

where $\mathbf{r}(t)=\hat{x} x(t)$ is the position vector of the bottom surface of the rubber block, and where $R$ is the radius of a macro-asperity contact region, which we assume to be circular. Using (14) and (16) gives

$$
\frac{\dot{Q}(z, t)}{\rho C_{V}}=\int_{0}^{\infty} \mathrm{d} q f(q, t) \mathrm{e}^{-2 q z} .
$$

Let us introduce a new coordinate system, moving with the bottom surface of the rubber block:

$$
\mathbf{x}^{\prime}=\mathbf{x}-\mathbf{r}(t) .
$$

Substituting this in (22), and replacing $\mathbf{x}^{\prime} \rightarrow \mathbf{x}$ for simplicity, gives

$$
\frac{\partial T}{\partial t}-\dot{\mathbf{r}}(t) \cdot \frac{\partial T}{\partial \mathbf{x}}-D \frac{\partial^{2} T}{\partial z^{2}}=\frac{\dot{Q}(z, t)}{\rho C_{V}} \theta(R-|\mathbf{x}|) .
$$

We now introduce the Fourier transform

$$
\begin{aligned}
& \tilde{T}(\mathbf{p}, k, t)=\frac{1}{(2 \pi)^{3}} \int \mathrm{d}^{2} x \mathrm{~d} z T(\mathbf{x}, z, t) \mathrm{e}^{-\mathrm{i}(\mathbf{p} \cdot \mathbf{x}+k z)} \\
& T(\mathbf{x}, z, t)=\int \mathrm{d}^{2} p \mathrm{~d} k \tilde{T}(\mathbf{p}, k, t) \mathrm{e}^{\mathrm{i}(\mathbf{p} \cdot \mathbf{x}+k z)} .
\end{aligned}
$$

Using (25) we get from (24)

$$
\frac{\partial \tilde{T}}{\partial t}-\mathbf{i p} \cdot \dot{\mathbf{r}}(t) \tilde{T}+D k^{2} \tilde{T}=\frac{\dot{Q}(k, t)}{\rho C_{V}} \frac{1}{2 \pi} \int_{0}^{R} \mathrm{~d} r r J_{0}(k r)
$$

where $J_{0}(x)$ is the zero order Bessel function and where

$$
\dot{Q}(k, t)=\frac{1}{2 \pi} \int \mathrm{d} z \dot{Q}(z, t) \mathrm{e}^{-\mathrm{i} k z} .
$$

Equation (27) is easy to integrate to get

$\tilde{T}(\mathbf{p}, k, t)=\int_{0}^{t} \mathrm{~d} t^{\prime} \mathrm{e}^{-D k^{2}\left(t-t^{\prime}\right)+\mathrm{i} p_{x}\left[x(t)-x\left(t^{\prime}\right)\right]} \frac{\dot{Q}\left(k, t^{\prime}\right)}{\rho C_{V}} \frac{1}{2 \pi} \int_{0}^{R} \mathrm{~d} r r J_{0}(k r)$. 
The laterally averaged temperature in the contact area is

$$
\begin{aligned}
\bar{T}(k, t)= & \frac{1}{\pi R^{2}} \int_{|\mathbf{x}|<R} \mathrm{~d}^{2} x T(\mathbf{x}, k, t) \\
& =\frac{1}{\pi R^{2}} \int_{|\mathbf{x}|<R} \mathrm{~d}^{2} x \int \mathrm{d}^{2} p \mathrm{e}^{\mathrm{i} \mathbf{p} \cdot \mathbf{x}} \tilde{T}(\mathbf{p}, k, t) \\
& =\frac{2}{R^{2}} \int \mathrm{d}^{2} p \int_{0}^{R} \mathrm{~d} r r J_{0}(k r) \tilde{T}(\mathbf{p}, k, t) .
\end{aligned}
$$

Substituting (29) in (30) gives after some simplifications

$$
\bar{T}(k, t)=\int_{0}^{t} \mathrm{~d} t^{\prime} \Gamma\left(t, t^{\prime}\right) \mathrm{e}^{-D k^{2}\left(t-t^{\prime}\right)} \frac{\dot{Q}\left(k, t^{\prime}\right)}{\rho C_{V}}
$$

where

$$
\Gamma\left(t, t^{\prime}\right)=\frac{2}{R^{2}} \int_{0}^{\infty} \mathrm{d} p p J_{0}\left(p\left[x(t)-x\left(t^{\prime}\right)\right]\right)\left(\int_{0}^{R} \mathrm{~d} r r J_{0}(p r)\right)^{2}
$$

Now since

we get with $p R=y$

$$
\int_{0}^{R} \mathrm{~d} r r J_{0}(p r)=\frac{R}{p} J_{1}(p R)
$$

$$
\Gamma\left(t, t^{\prime}\right)=2 \int_{0}^{\infty} \mathrm{d} y y^{-1} J_{0}(2 w y)\left(J_{1}(y)\right)^{2}=h(w)
$$

where

$$
w=w\left(t, t^{\prime}\right)=\left[x(t)-x\left(t^{\prime}\right)\right] / 2 R .
$$

Performing the integral in (32) gives

$$
h(w)=1-\frac{2}{\pi} w\left(1-w^{2}\right)^{1 / 2}-\frac{2}{\pi} \arcsin (w)
$$

for $w<1$ while $h=0$ for $w>1$. Using (23) we get

$$
\frac{\dot{Q}(k, t)}{\rho C_{V}}=\frac{1}{2 \pi} \int_{0}^{\infty} \mathrm{d} q f(q, t) \frac{4 q}{k^{2}+4 q^{2}} .
$$

Next, using that

$$
\bar{T}_{q}(t)=2 q \int_{0}^{\infty} \mathrm{d} z \bar{T}(z, t) \mathrm{e}^{-2 q z}=2 \int_{0}^{\infty} \mathrm{d} k \frac{4 q^{2}}{k^{2}+4 q^{2}} \bar{T}(k, t)
$$

we get from (31), (32), (35) and (36)

$\bar{T}_{q}(t)=T_{0}+\int_{0}^{t} \mathrm{~d} t^{\prime} \Gamma\left(t, t^{\prime}\right) \int_{0}^{\infty} \mathrm{d} q^{\prime} f\left(q^{\prime}, t^{\prime}\right) \frac{1}{\pi} \int_{0}^{\infty} \mathrm{d} k \frac{4 q^{2}}{k^{2}+4 q^{2}} \frac{4 q^{\prime}}{k^{2}+4 q^{\prime 2}} \mathrm{e}^{-D k^{2}\left(t-t^{\prime}\right)}$.

Let us summarize the basic equations derived above. The friction coefficient

$\mu(t) \approx \frac{1}{2} \int_{q_{0}}^{q_{1}} \mathrm{~d} q q^{3} C(q) P(q, t) \int_{0}^{2 \pi} \mathrm{d} \phi \cos \phi \operatorname{Im} \frac{E\left(q v(t) \cos \phi, T_{q}(t)\right)}{\left(1-v^{2}\right) \sigma_{0}}$

where $\sigma_{0}=F_{\mathrm{N}} / A_{0}$, where $A_{0}$ is the nominal contact area. In this equation enters the flash temperature at time $t$ :

$T_{q}(t)=T_{0}+\int_{0}^{t} \mathrm{~d} t^{\prime} \Gamma\left(t, t^{\prime}\right) \int_{0}^{\infty} \mathrm{d} q^{\prime} f\left(q^{\prime}, t^{\prime}\right) \frac{1}{\pi} \int_{0}^{\infty} \mathrm{d} k \frac{4 q^{2}}{k^{2}+4 q^{2}} \frac{4 q^{\prime}}{k^{2}+4 q^{\prime 2}} \mathrm{e}^{-D k^{2}\left(t-t^{\prime}\right)}$ 
where $\Gamma\left(t, t^{\prime}\right)=h\left(w\left(t, t^{\prime}\right)\right)$ with $w\left(t, t^{\prime}\right)=\left[x(t)-x\left(t^{\prime}\right)\right] / 2 R$ depend on the history of the sliding motion. The function

$$
f(q, t)=\frac{v(t)}{\rho C_{v}} q^{4} C(q) \frac{P(q, t)}{P\left(q_{\mathrm{m}}, t\right)} \int \mathrm{d} \phi \cos \phi \operatorname{Im} \frac{E\left(q v(t) \cos \phi, T_{q}(t)\right)}{1-v^{2}},
$$

where $v=\dot{x}(t)$ depends on time. The function $P(q, t)$ (which also depends on time) is given by the standard formula

$$
P(q, t)=\frac{2}{\pi} \int_{0}^{\infty} \mathrm{d} x \frac{\sin x}{x} \exp \left[-x^{2} G(q, t)\right]=\operatorname{erf}\left(\frac{1}{2 \sqrt{ } G}\right)
$$

where

$$
G(q, t)=\frac{1}{8} \int_{q_{0}}^{q} \mathrm{~d} q q^{3} C(q) \int_{0}^{2 \pi} \mathrm{d} \phi\left|\frac{E\left(q v(t) \cos \phi, T_{q}(t)\right)}{\left(1-v^{2}\right) \sigma_{0}}\right|^{2}
$$

\subsection{Limiting cases and physical interpretation of $h(w)$.}

When the heat diffusivity $D=0$, equation (39) takes the form

$$
\bar{T}_{q}(t)=T_{0}+\int_{0}^{t} \mathrm{~d} t^{\prime} \Gamma\left(t, t^{\prime}\right) \int_{0}^{\infty} \mathrm{d} q^{\prime} f\left(q^{\prime}, t^{\prime}\right) \frac{q}{q+q^{\prime}} .
$$

In the limit $D \rightarrow \infty$ we get, as expected, $T=T_{0}$.

Let us consider stationary sliding so that $x(t)=v_{0} t$. In this case $w=\left(v_{0} / 2 R\right)\left(t-t^{\prime}\right)$ and $f(q, t)=f(q)$ is independent of time. Thus (39) takes the form

$$
\bar{T}_{q}(t) \approx T_{0}+\int_{0}^{\infty} \mathrm{d} q^{\prime} G\left(q, q^{\prime}\right) f\left(q^{\prime}\right)
$$

where

$$
G\left(q, q^{\prime}\right)=\frac{1}{\pi} \int_{0}^{\infty} \mathrm{d} k \frac{4 q^{2}}{k^{2}+4 q^{2}} \frac{4 q^{\prime}}{k^{2}+4 q^{\prime 2}} M(k)
$$

where

$$
M(k)=\int_{t-2 R / v_{0}}^{t} \mathrm{~d} t^{\prime} h(w) \mathrm{e}^{-D k^{2}\left(t-t^{\prime}\right)} .
$$

Changing the integration variable from $t^{\prime}$ to $w$ gives

$$
M(k)=\frac{2 R}{v_{0}} \int_{0}^{1} \mathrm{~d} w h(w) \mathrm{e}^{-\left(2 R / v_{0}\right) D k^{2} w} .
$$

The result (45) is the same as (21) but with a slightly different (and more accurate) function $M(k)$. Thus, in (21) there occurs instead of $M(k)$ the factor

$$
\frac{1}{D k^{2}}\left(1-\mathrm{e}^{-D k^{2} t_{0}}\right)=t_{0} \int_{0}^{1} \mathrm{~d} w \mathrm{e}^{-t_{0} D k^{2} w} .
$$

Since $t_{0}$ is of order $R / v_{0}$ and $h(w)$ of order unity, these two expressions for the steady rubber friction are of similar magnitude.

Here I present an alternative derivation of the factor $h(w)=g\left(t, t^{\prime}\right)$, which gives a direct physical interpretation of this factor. In the theory above this function emerged purely mathematically, as a result of an integral of a product of three Bessel functions.

Let us assume that the heat diffusivity $D=0$ and consider the equation

$$
\frac{\partial T}{\partial t}=K(z, t) \theta(R-|\mathbf{x}-\mathbf{r}(t)|)
$$




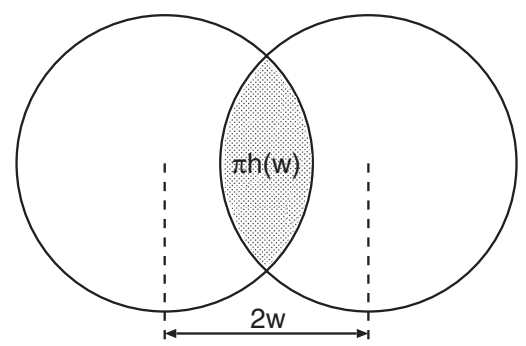

Figure 10. The common region (dotted area) between the two circles has the area $\pi h(w)$.

where $K=\dot{Q} / \rho C_{V}$ and where $\theta(x)$ is the step function. Here $R$ is the radius of the macrocontact area and $\mathbf{r}(t)$ the position vector of the contact area. Integrating (48) gives

$$
T(\mathbf{x}, z, t)=T_{0}+\int_{0}^{t} \mathrm{~d} t^{\prime} \theta\left(R-\left|\mathbf{x}-\mathbf{r}\left(t^{\prime}\right)\right|\right) K\left(z, t^{\prime}\right) .
$$

Averaging the temperature (49) over the contact area gives

$$
\begin{aligned}
\bar{T}(z, t)=T_{0} & +\frac{1}{\pi R^{2}} \int_{|\mathbf{x}-\mathbf{r}(t)|<R} \mathrm{~d}^{2} x \int_{0}^{t} \mathrm{~d} t^{\prime} \theta\left(R-\left|\mathbf{x}-\mathbf{r}\left(t^{\prime}\right)\right|\right) K\left(z, t^{\prime}\right) \\
& =T_{0}+\frac{1}{\pi R^{2}} \int_{0}^{t} \mathrm{~d} t^{\prime} K\left(z, t^{\prime}\right) \int_{|\mathbf{x}|<R} \mathrm{~d}^{2} x \theta\left(R-\left|\mathbf{x}+\mathbf{r}(t)-\mathbf{r}\left(t^{\prime}\right)\right|\right) .
\end{aligned}
$$

If we change integration variable $\mathbf{x}^{\prime}=\mathbf{x} / R$ and denote $\mathbf{x}^{\prime}$ with $\mathbf{x}$ for simplicity, we get from (50)

$$
\bar{T}=T_{0}+\frac{1}{\pi} \int_{0}^{t} \mathrm{~d} t^{\prime} K\left(z, t^{\prime}\right) \int_{|\mathbf{x}|<1} \mathrm{~d}^{2} x \theta(1-|\mathbf{x}+2 \mathbf{w}|)
$$

where

$$
\mathbf{w}=\left[\mathbf{r}(t)-\mathbf{r}\left(t^{\prime}\right)\right] / 2 R .
$$

Note that the integral

$$
h(w)=\frac{1}{\pi} \int_{|\mathbf{x}|<1} \mathrm{~d}^{2} x \theta(1-|\mathbf{x}+2 \mathbf{w}|)
$$

is the common area (divided by $\pi$ ) between two circular regions (with unit radius) with the origins separated by the distance $2 w$; see figure 10 . It is easy to calculate

$$
h(w)=1-\frac{2}{\pi} w\left(1-w^{2}\right)^{1 / 2}-\frac{2}{\pi} \arcsin (w)
$$

for $w<1$ while $h(w)=0$ for $w>1$. This formula agrees with (34). Note that $w=w\left(t, t^{\prime}\right)$ and $h(w)=\Gamma\left(t, t^{\prime}\right)$ so that (51)-(53) gives

$$
\bar{T}=T_{0}+\frac{1}{\pi} \int_{0}^{t} \mathrm{~d} t^{\prime} \Gamma\left(t, t^{\prime}\right) K\left(z, t^{\prime}\right) .
$$

Finally, using (14), (16) and (19) in (54) gives

$$
\bar{T}_{q}(t)=T_{0}+\int_{0}^{t} \mathrm{~d} t^{\prime} \Gamma\left(t, t^{\prime}\right) \int_{0}^{\infty} \mathrm{d} q^{\prime} f\left(q^{\prime}, t^{\prime}\right) \frac{q}{q+q^{\prime}}
$$

which is the same as (43). Thus, we conclude that the factor $h(w)=\Gamma\left(t, t^{\prime}\right)$ in (37) is equal to the overlap area (divided by $\pi$ ) between the contact area at time $t$, and the contact area at an earlier time $t^{\prime}$, see figure 10 . 


\section{Sliding dynamics: numerical results}

In this section we will present numerical results for the friction force acting on a rubber (tyre tread) block, sliding on an asphalt road surface. We will study the friction as a function of the velocity of the bottom surface of the block. In real experiments it is, of course, not possible to specify the motion of the bottom surface directly (unless steady sliding occurs), but usually one specifies the motion of the top surface of the rubber block (or some other distant part of the system). We will consider this case later. In this section we assume the rubber background temperature $T_{0}=60^{\circ} \mathrm{C}$, the substrate is an asphalt road surface (with the power spectrum given in figure 2), and include the substrate roughness down to the wavevector cut-off $q_{1}=\zeta_{\max } q_{0}$ with $\zeta_{\max }=400$ and $q_{0}=1500 \mathrm{~m}^{-1}$ so that $q_{1}=6 \times 10^{5}$ or $\lambda_{1}=2 \pi / q_{1} \approx 10 \mu \mathrm{m}$. All calculations are for the nominal (squeezing) pressure $\sigma_{0}=0.4 \mathrm{MPa}$.

\subsection{Stationary motion}

Figure 11 shows the friction coefficient (a), the flash temperature (b) and the logarithm of the (normalized) area of contact (c), as a function of the logarithm of the sliding velocity. In (a) we show the kinetic friction coefficient both with and without the flash temperature. In (b) and (c) we show results both for $\zeta=1$ and at the highest magnification $\zeta=400$. Note that without the flash temperature the rubber friction coefficient increases monotonically up to the highest studied sliding velocity $v \approx 100 \mathrm{~m} \mathrm{~s}^{-1}$. When the flash temperature is included in the study the friction is maximal for $v \approx 1 \mathrm{~cm} \mathrm{~s}^{-1}$. The decrease of the friction observed when the flash temperature is included in the analysis is easy to understand (see also section 4): when the rubber heats up the viscoelastic modulus $E(\omega)$ shifts towards higher frequencies and the rubber becomes more elastic (less viscous), resulting in less energy dissipation.

Figure 11(b) shows that the flash temperature at the magnification $\zeta=400$ (i.e. the temperature about $10 \mu \mathrm{m}$ below the rubber surface) is $\approx 140{ }^{\circ} \mathrm{C}$, i.e. about $80^{\circ} \mathrm{C}$ above the rubber background temperature. On the other hand the temperature increase a few millimetres below the surface (corresponding to the magnification $\zeta=1$ ) is just a few degrees. We note that during steady sliding the temperature in the whole rubber block will increase continuously with increasing time, but this effect is not included in the discussion above, unless one allows for the background temperature $T_{0}$ to increase with increasing time. The calculation of the time dependence of $T_{0}$ requires the knowledge of the temperature of the surrounding medium, and the heat transfer coefficient to the surroundings. This topic is important in many practical applications, but does not interest us here.

Figure 11(c) shows that the area of (apparent) contact at low magnification $(\zeta=1)$ decreases continuously with increasing sliding velocity. This result is expected because the frequencies $\omega$ of the pulsating deformations of the rubber increase with increasing sliding velocity $v(\omega \sim v)$, and rubber becomes elastically stiffer at higher frequencies, resulting in an (apparent) smaller contact area at high sliding velocity. However, at the highest magnification $\zeta=400$, figure 11(c) shows that the area of contact increases with increasing sliding velocity for $v>0.1 \mathrm{~m} \mathrm{~s}^{-1}$. This is caused by the increase in the temperature in the surface region of the rubber, which shifts the viscoelastic modulus towards higher frequencies faster than the linear increase $\omega \sim v$ in the perturbing frequencies arising from the increase in the sliding velocity.

Figure 12 shows (a) the friction coefficient, (b) the flash temperature, and (c) the logarithm of the (normalized) area of contact, as a function of the logarithm of the magnification, for the sliding velocity $v=1 \mathrm{~m} \mathrm{~s}^{-1}$. In (a) and (c) we show results both with and without the flash temperature. Figure 12(a) shows that when the flash temperature is included the surface roughness on every decade in length scale contributes roughly with an equal amount to the 

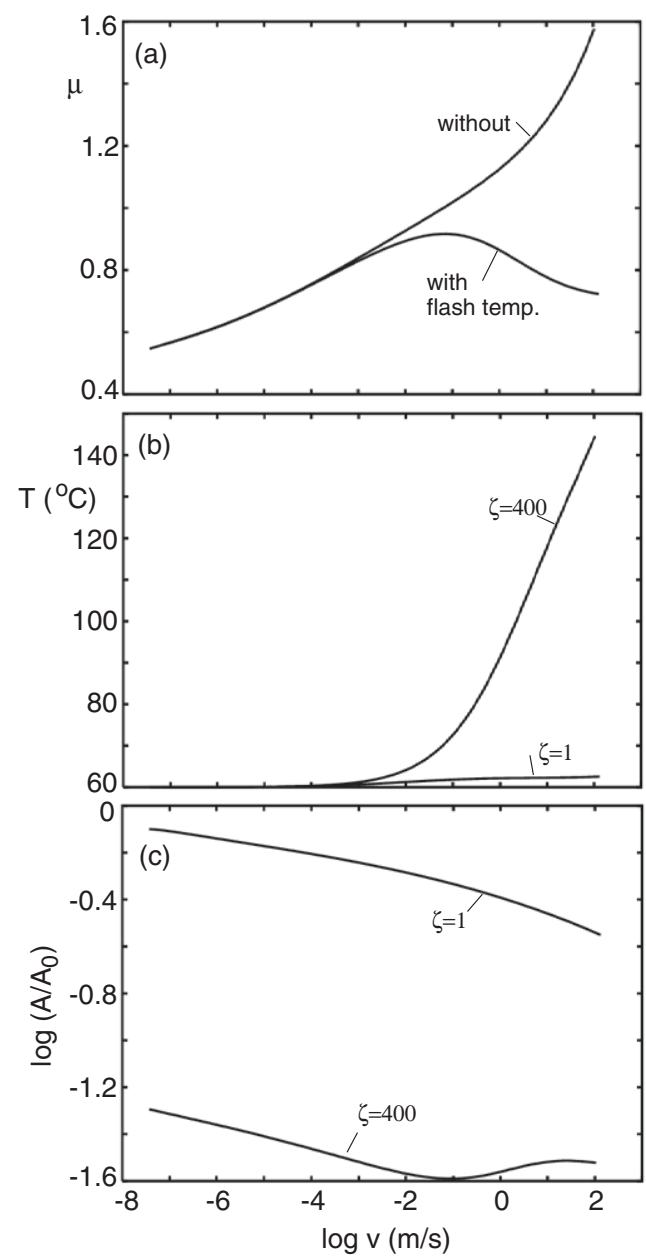

Figure 11. The friction coefficient (a), the flash temperature (b), and the logarithm of the (normalized) area of contact (c), as a function of the logarithm of the sliding velocity, for a rubber block (tyre tread rubber) sliding on an asphalt road surface. The background temperature $T_{0}=60^{\circ} \mathrm{C}$ and $\zeta_{\max }=400$. In (a) we show the kinetic friction coefficient both with and without the flash temperature. In (b) and (c) we show results both for $\zeta=1$ and at the highest magnification $\zeta=400$.

rubber sliding friction. Figure 12(c) shows that at low magnification the contact area is the same with and without the flash temperature included in the analysis, while for large magnification the area of contact increases when the flash temperature is included, as expected from the elastic softening of the rubber at higher temperatures (see above).

\subsection{Non-stationary motion}

It is usually assumed in simple treatments of friction that the friction coefficient is a function only of the instantaneous slip velocity $v_{\mathrm{b}}(t)$, i.e. $\mu(t)=\mu\left(v_{\mathrm{b}}(t)\right)$, where $v_{\mathrm{b}}(t)$ is the velocity of the bottom surface (in contact with the substrate) of the sliding block. However, this approximation fails badly for rubber friction. The reason is the strong dependence of rubber friction on the temperature distribution in the rubber block. Since the temperature distribution 

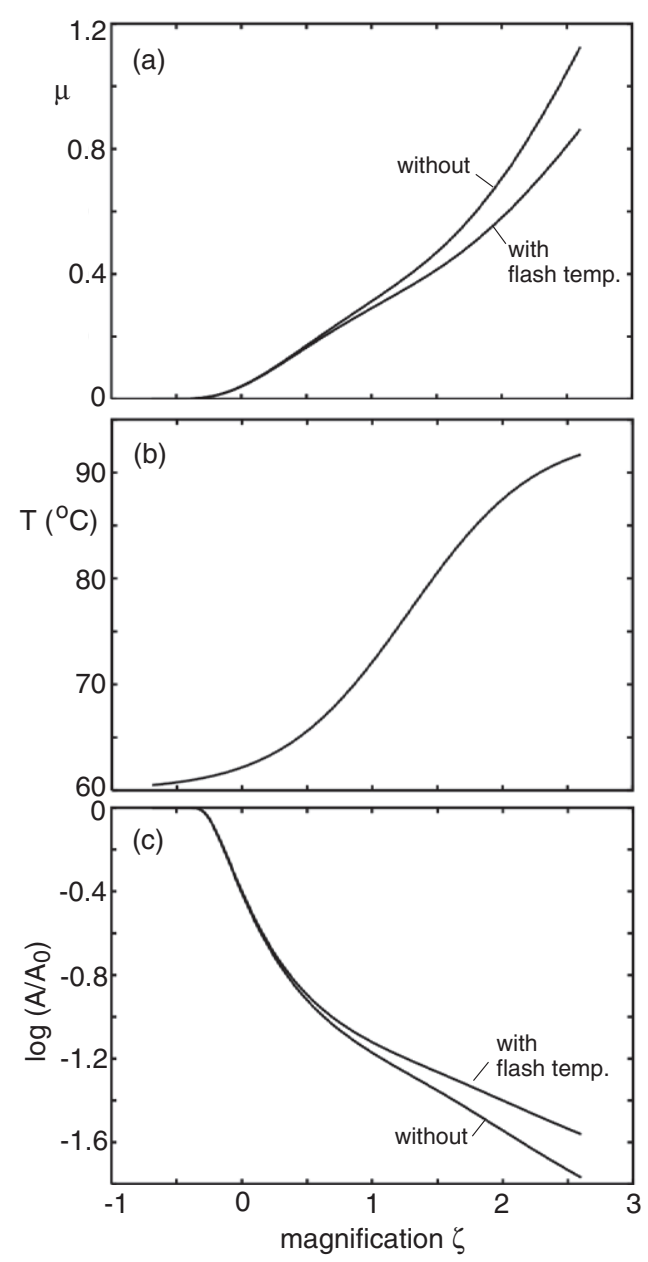

Figure 12. The friction coefficient (a), the flash temperature (b), and the logarithm of the (normalized) area of contact (c), as a function of the logarithm of the magnification, for a rubber block (tyre tread rubber) sliding on an asphalt road surface. The background temperature $T_{0}=60^{\circ} \mathrm{C}$ and the sliding velocity $v=1 \mathrm{~m} \mathrm{~s}^{-1}$. In (a) and (c) we show results both with and without the flash temperature.

$T(\mathbf{x}, t)$ at time $t$ depends on the sliding motion for all earlier times $t^{\prime} \leqslant t$, it follows immediately that for rubber-like materials the friction coefficient will depend on the sliding history, i.e. it will be a functional of $v_{\mathrm{b}}(t): \mu=\mu\left(v_{\mathrm{b}}\left(t^{\prime}\right), t^{\prime} \leqslant t\right)$. In this section I present numerical results which illustrate this fact.

Let us assume the velocity of the bottom surface of the block first increases linearly with time from zero to $1 \mathrm{~m} \mathrm{~s}^{-1}$ and then decreases back to zero, as indicated in figure 13(a). In case 1 the time for the whole cycle is $0.002 \mathrm{~s}$ and for case $20.004 \mathrm{~s}$. In figure 13(b) we show the resulting friction coefficient. Note that $\mu$ exhibits hysteresis as a function of the sliding velocity, and that $\mu$ is smaller during the retardation than during the acceleration time period. This behaviour just reflects the finite slip distance necessary for building up the flash temperature, and the finite time involved in heat diffusion, and shows that $\mu$ does not just depend on the instantaneous sliding velocity but on the whole sliding history (memory effects). Note that the slip distance is given by $v_{\max }^{2} / a$, where $v_{\max }=1 \mathrm{~m} \mathrm{~s}^{-1}$ is the maximum of the 

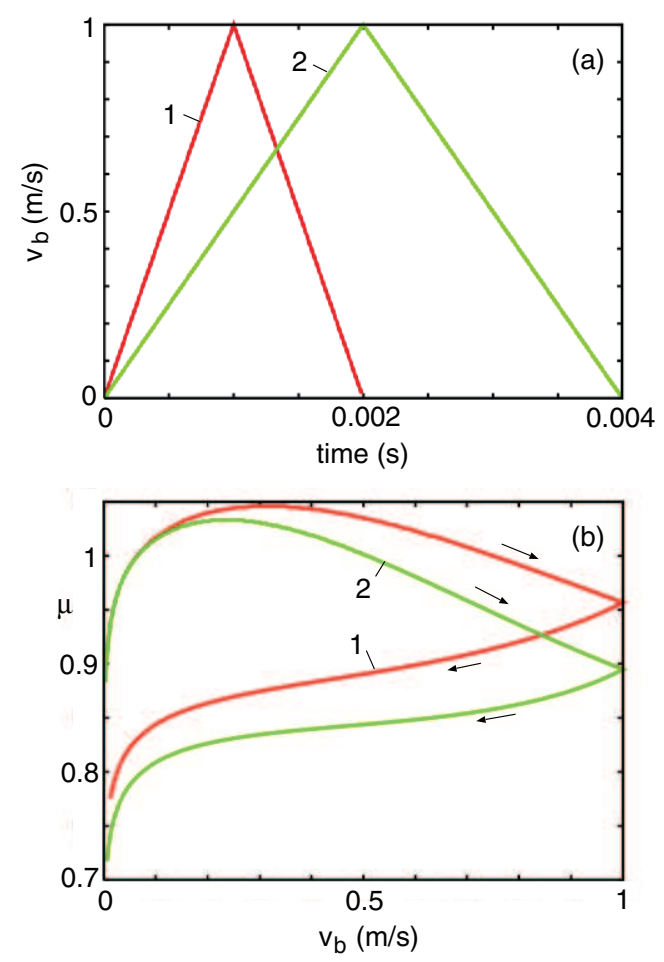

Figure 13. Non-uniform sliding motion. (a) The velocity of the bottom surface of the block increases linearly with time from zero to $1 \mathrm{~m} \mathrm{~s}^{-1}$ and then decreases back to zero. In case 1 the time for the whole velocity cycle is $0.002 \mathrm{~s}$ and for case $20.004 \mathrm{~s}$. (b) The friction coefficient exhibits hysteresis as a function of the sliding velocity, and is smaller during the retardation time period than during the acceleration time period. This behaviour reflects the build-up of the flash temperature during the sliding cycle.

velocity and the acceleration $a=1000$ and $500 \mathrm{~m} \mathrm{~s}^{-2}$ for case $\mathbf{1}$ and $\mathbf{2}$ respectively, giving the slip distances 1 and $2 \mathrm{~mm}$, respectively. These values are both smaller than the diameter of the macro-asperity contact regions, which is $2 R \approx 3.3 \mathrm{~mm}$. The larger slip distance for case 2 implies that more energy is deposited (in the rubber) at the rubber-substrate contact regions than for case $\mathbf{1}$, which will result in a higher flash temperature in case $\mathbf{2}$ than in case $\mathbf{1}$. This explains why the friction coefficient is smaller for case $\mathbf{2}$ (see figure 13(b)).

The temperature build-up during the sliding motion is illustrated in figure 14. This figure also shows that after the sliding motion has stopped, i.e. for $t>0.002 \mathrm{~s}$ and $t>0.004 \mathrm{~s}$ for cases $\mathbf{1}$ and 2, respectively, the temperature at the surface decreases monotonically because of heat diffusion. Note that as a function of the slip velocity $v_{\mathrm{b}}$ (not shown) the flash temperature in figure 14(a) is always higher for case 2 as compared to case 1. Figure 14(b) shows the frictional shear stress $\sigma_{\mathrm{f}}(t)=\sigma_{0} \mu(t)$ as a function of time.

Assume now that the bottom surface of the block moves with the constant velocity $v_{\mathrm{b}}=2 \mathrm{~m} \mathrm{~s}^{-1}$ for $0<t<0.002 \mathrm{~s}$, and at $t=0.002 \mathrm{~s}$ it is abruptly reduced to $10^{-5} \mathrm{~m} \mathrm{~s}^{-1}$, and is then kept at this value for $0.0005 \mathrm{~s}$ (case 1), $0.001 \mathrm{~s}$ (case 2), $0.002 \mathrm{~s}$ (case 3), $0.003 \mathrm{~s}$ (case 4), and then returned to $v_{\mathrm{b}}=2 \mathrm{~m} \mathrm{~s}^{-1}$. For this sliding history we show in figure 15 (a) the friction coefficient, (b) the flash temperature at the highest magnification and (c) the (relative) contact area at the highest magnification, as a function of time. Note that the longer the system is kept in the low-velocity state (where $v=10^{-5} \mathrm{~m} \mathrm{~s}^{-1}$ ), the higher the 'start-up' 


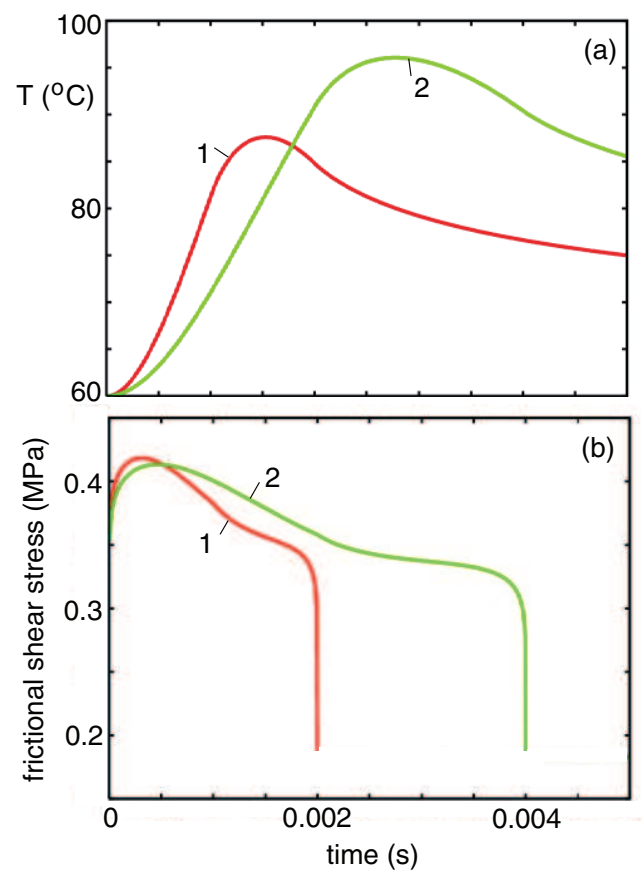

Figure 14. The flash temperature (a) and the frictional shear stress (b) as a function of time as the bottom surface of the rubber block performs the motion indicated in figure 13(a), and with $v_{\mathrm{b}}=0$ for $t>0.002 \mathrm{~s}$ and $0.004 \mathrm{~s}$ for cases $\mathbf{1}$ and $\mathbf{2}$, respectively.

peak in the friction will be when the velocity is switched back to $2 \mathrm{~m} \mathrm{~s}^{-1}$. The reason for this is that, because of heat diffusion, in the low-velocity state the temperature in the rubber will decrease continuously with increasing time, see figure 15(b), and the rubber friction increases when the rubber temperature decreases. Figure 15(c) illustrates that, as expected, the contact area between the rubber and the substrate increases when the rubber heats up and when the rubber sliding velocity decreases.

\section{Rubber block dynamics}

We consider the simplest but most fundamental case of a rubber block (mass $M$ ) squeezed against a substrate, and with the upper surface clamped and in prescribed lateral motion as indicated in figure 16.

The shear stress that acts on the lower surface of the rubber block is treated as a spatially uniform stress $\sigma_{\mathrm{f}}(t)$. In the most general case, where inertia effects are important, we discretize the block (thickness $d$ ) into $N$ layers of thickness $\Delta d=d / N$ and mass $m=M / N$. The coordinates $x_{1}(t)=x_{\mathrm{b}}(t), x_{2}(t), \ldots, x_{N}(t), x_{N+1}(t)=x_{\mathrm{t}}(t)$ depend on time. The continuum limit is obtained as $N \rightarrow \infty$. The non-stationary sliding of the rubber block (see figure 16) is determined by the equations of motion

$$
\begin{aligned}
& m \ddot{x}_{b}(t)=F_{1}(t)-F_{0}\left[x_{b}(t)\right], \\
& m \ddot{x}_{2}(t)=F_{2}(t)-F_{1}(t), \\
& m \ddot{x}_{3}(t)=F_{3}(t)-F_{2}(t), \\
& \cdots \\
& m \ddot{x}_{N}(t)=F_{N}(t)-F_{N-1}(t),
\end{aligned}
$$




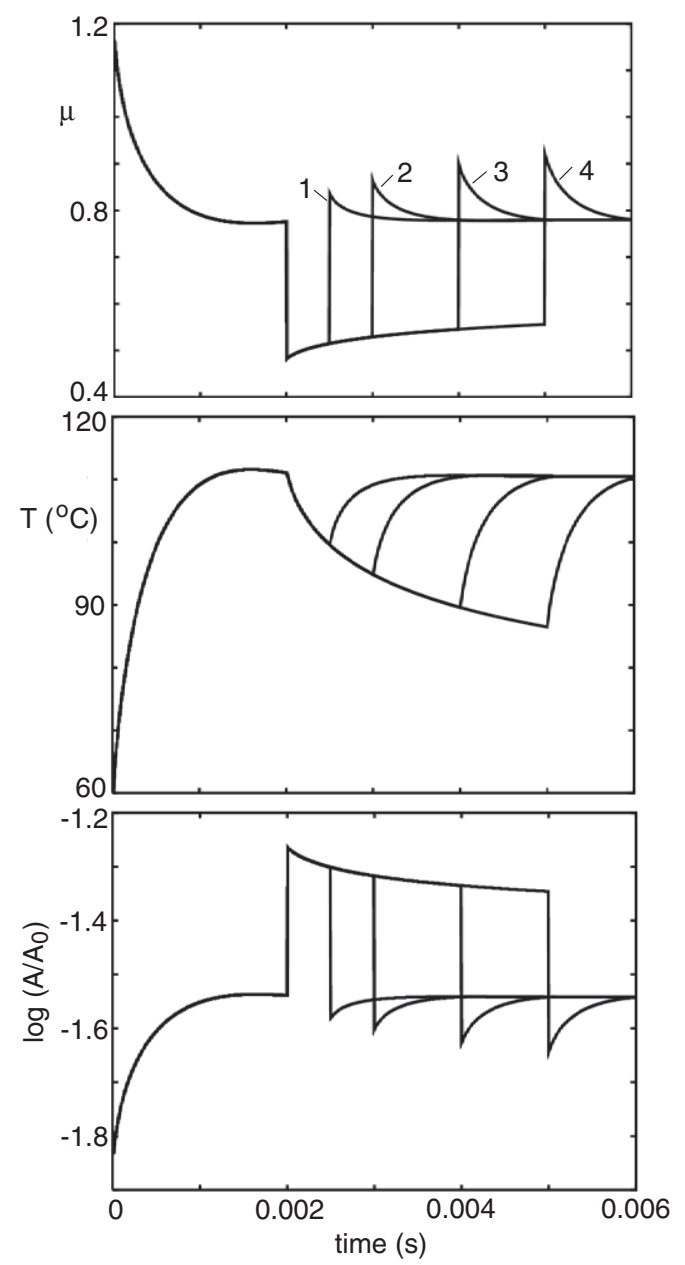

Figure 15. (a) The friction coefficient, (b) the flash temperature at the highest magnification and (c) the (relative) contact area at the highest magnification, as a function of time. The bottom surface of the block moves with the constant velocity $v_{\mathrm{b}}=2 \mathrm{~m} \mathrm{~s}^{-1}$ for $0<t<0.002 \mathrm{~s}$, and at $t=0.002 \mathrm{~s}$ it is abruptly reduced to $10^{-5} \mathrm{~m} \mathrm{~s}^{-1}$ and is kept at this velocity for $0.0005 \mathrm{~s}$ (case 1), $0.001 \mathrm{~s}$ (case 2), $0.002 \mathrm{~s}$ (case 3), $0.003 \mathrm{~s}$ (case 4), and then returned to $v_{\mathrm{b}}=2 \mathrm{~m} \mathrm{~s}^{-1}$.

where $F_{0}$ is the friction force acting on the bottom surface of the block, and $F_{i}$ the shear force acting on layer $i$ from the layer $i+1$ above. $F_{N}$ is the force acting on layer $N$ from the drive (the black slab on top of the rubber block in figure 16).

Note that the friction force $F_{0}\left[x_{b}(t)\right]$ is a functional of $x_{b}(t)$ since it depends on the whole history $\left\{x_{b}\left(t^{\prime}\right) ; t^{\prime} \leqslant t\right\}$. The shear force $F_{i}$ acting on layer $i$ from the layer above can be derived from the equation relating the shear stress $\sigma(\omega)$ to the shear strain $\epsilon(\omega)$ via

$$
\sigma(\omega)=G(\omega) \epsilon(\omega)
$$

where $G(\omega)$ is the complex frequency dependent shear modulus. In our case $(i=1, \ldots, N)$

$$
\epsilon(t)=\left[x_{i+1}(t)-x_{i}(t)\right] / \Delta d,
$$

where $\Delta d$ is the thickness of the rubber layer. If we multiply (56) with the area $A_{0}=L_{x} L_{y}$ of 


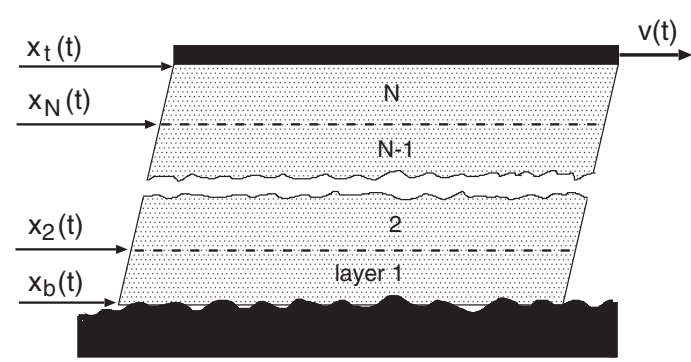

Figure 16. A rubber block (thickness $d$ ) pulled on a rough hard substrate. The upper surface is clamped and moved with a prescribed velocity $v(t)=\dot{x}_{\mathrm{t}}$. In the mathematical description of the sliding motion, the block is discretized into $N$ layers of thickness $\Delta d=d / N$. The coordinates $x_{1}(t)=x_{\mathrm{b}}(t), x_{2}(t), \ldots, x_{N}(t), x_{N+1}=x_{\mathrm{t}}(t)$ depend on time. The continuum limit is obtained as $N \rightarrow \infty$.

the tread block we get the shear force

$$
F_{i}(\omega)=A_{0} G(\omega) \epsilon(\omega),
$$

or in time-space

$$
F_{i}(t)=\int \mathrm{d} \omega F_{i}(\omega) \mathrm{e}^{-\mathrm{i} \omega t}=\int \mathrm{d} \omega A_{0} G(\omega) \epsilon(\omega) \mathrm{e}^{-\mathrm{i} \omega t} .
$$

Substituting

$$
\epsilon(\omega)=\frac{1}{2 \pi} \int \mathrm{d} t^{\prime} \epsilon\left(t^{\prime}\right) \mathrm{e}^{\mathrm{i} \omega t^{\prime}}
$$

in equation (58) gives

$$
F_{i}(t)=\int_{-\infty}^{\infty} \mathrm{d} t^{\prime} A_{0} G\left(t-t^{\prime}\right) \epsilon\left(t^{\prime}\right),
$$

where

$$
G(t)=\frac{1}{2 \pi} \int_{-\infty}^{\infty} \mathrm{d} \omega G(\omega) \mathrm{e}^{-\mathrm{i} \omega t} .
$$

If we assume that $\epsilon(t)=0$ for $t<0$ and if we use that $G(t)$ must vanish for $t<0$ because of causality (see section 7), we get

$$
F_{i}(t)=\int_{0}^{t} \mathrm{~d} t^{\prime} A_{0} G\left(t-t^{\prime}\right) \epsilon\left(t^{\prime}\right),
$$

or, if we use (57),

$$
F_{i}(t)=\int_{0}^{t} \mathrm{~d} t^{\prime} K\left(t-t^{\prime}\right)\left[x_{i+1}\left(t^{\prime}\right)-x_{i}\left(t^{\prime}\right)\right],
$$

and in particular

$$
F_{N}(t)=\int_{0}^{t} \mathrm{~d} t^{\prime} K\left(t-t^{\prime}\right)\left[x_{\mathrm{t}}\left(t^{\prime}\right)-x_{N}\left(t^{\prime}\right)\right],
$$

where the memory spring

$$
K(t)=A_{0} G(t) / \Delta d
$$

The equations above can also be applied when the properties of the rubber block depends on the vertical coordinate $z$ if one replaces the mass $m \rightarrow m_{i}$ and memory spring $K \rightarrow K_{i}$. 
If $\rho=\rho(z)$ is the rubber mass density, then $m_{i}=A_{0} \Delta d \rho\left(z_{i}\right)$ and similarly $K_{i}=$ $A_{0} G\left(z_{i}, t\right) / \Delta d$. Since the rubber friction $F_{0}$ depends extremely strongly on the sliding velocity for very small sliding velocities, the system of differential equations given above is of the stiff nature, which requires special care in the time integration in order to avoid numerical instabilities.

\section{Viscoelastic modulus}

Many experimental techniques can be used to obtain the complex, frequency dependent, shear modulus $G(\omega)$ (or Young modulus $E(\omega)$ ). This quantity enters directly in the calculation of the rubber frictional shear stress $\sigma_{\mathrm{f}}$. But in the rubber block dynamics theory described in section 6 we also need the time-dependent real shear modulus

$$
G(t)=\frac{1}{2 \pi} \int_{-\infty}^{\infty} \mathrm{d} \omega G(\omega) \mathrm{e}^{-\mathrm{i} \omega t}
$$

Since $G(\omega)$ usually is known only in a finite frequency interval, $\omega_{0}<\omega<\omega_{1}$, it is not easy to calculate $G(t)$ directly from (65) using, e.g., the fast Fourier transform method. Another, even more serious problem is that in some cases $G(t)$ is not a 'perfect' linear response function (see below), which gives rise to serious problems if one tries to calculate $G(t)$ directly from (65); e.g., one finds that $G(t)$ is complex rather than real.

Let us first assume that $G(\omega)$ is the viscoelastic modulus of a linear viscoelastic solid. Now, the shear stress $\sigma(t)$ in a solid at time $t$ can only depend on the deformations (or strain) the solid has undergone at earlier times, i.e. it cannot depend on the future strain (causality). Thus we can write

$$
\sigma(t)=\int_{-\infty}^{t} \mathrm{~d} t^{\prime} G\left(t-t^{\prime}\right) \epsilon\left(t^{\prime}\right)
$$

The Fourier transform of this equation gives

$$
\sigma(\omega)=G(\omega) \epsilon(\omega)
$$

where

$$
G(\omega)=\int_{0}^{\infty} \mathrm{d} t G(t) \mathrm{e}^{\mathrm{i} \omega t} .
$$

Since $\operatorname{Re}(\mathrm{i} \omega t)<0$ for $t>0$ and $\operatorname{Im}(\omega)>0$ it follows that $G(\omega)$ is an analytical function of $\omega$ in the upper half of the complex frequency plane. Thus all the singularities (poles and branch cuts) of $G(\omega)$ must occur in the lower part of the complex $\omega$-plane and we may write

$$
G(\omega)=G_{\infty}-\int_{0}^{\infty} \mathrm{d} \tau \frac{H(\tau)}{1-\mathrm{i} \omega \tau}
$$

where the spectral density $H(\tau)$ is a real positive function of the relaxation time $\tau$. This representation of $G(\omega)$ has the correct analytical properties (all the singularities occur in the lower part of the complex $\omega$-plane, as required by causality). The idea is now to determine $G_{\infty}$ and $H(\omega)$ so that the difference $\Delta G(\omega)=G_{\text {meas }}(\omega)-G(\omega)$ between the measured $G_{\text {meas }}$ shear modulus and the analytical expression $G(\omega)$ given by (66) becomes as small as possible. Thus, we minimize the quantity

$$
V=\frac{1}{\omega_{1}-\omega_{0}} \int_{\omega_{0}}^{\omega_{1}} \mathrm{~d} \omega \frac{|\Delta G(\omega)|}{\left|G_{\text {meas }}(\omega)\right|} .
$$

Note that

$$
\Delta G=G_{\text {meas }}(\omega)-G_{\infty}+\int_{0}^{\infty} \mathrm{d} \tau \frac{H(\tau)}{1-\mathrm{i} \omega \tau}
$$


In practice, $G_{\text {meas }}$ is only known at a set of discrete frequencies $\omega_{n}, n=1,2, \ldots, N$. Thus, (67) must be replaced with

$$
V=\frac{1}{N} \sum_{n=1}^{N} \frac{\left|\Delta G\left(\omega_{n}\right)\right|}{\left|G_{\text {meas }}\left(\omega_{n}\right)\right|} .
$$

Furthermore, in numerical calculations it is only possible to include a finite number of relaxation times $\tau_{k}$ in the spectral representation of $G(\omega)$ and write

$$
G(\omega) \approx G_{\infty}-\sum_{k} \frac{H_{k}}{1-\mathrm{i} \omega \tau_{k}} .
$$

where

$$
H(\tau) \approx \sum_{k} H_{k} \delta\left(\tau-\tau_{k}\right)
$$

If the relaxation times are distributed exponentially (i.e. $\tau_{k}=\tau_{0} \exp \left(\eta_{k}\right)$, where $\eta_{k}$ are uniformly distributed), it is usually enough to include $\sim 10-15$ relaxation times, or one relaxation time for each decade in frequency. If the measured $G_{\text {meas }}(\omega), \omega_{0}<\omega<\omega_{1}$, correspond to a true linear response function then one can find $G_{\infty}$ and $H(\omega)$ so that $V$ vanish. Since in many cases $G(\omega)$ is not a true linear response function (see appendix B) it is impossible to choose the real function $H(\omega)$ so that $V$ vanish. However, if we can find $H(\omega)$ and $G_{\infty}$ so that $V \ll 1$ we have a very good representation of $G(\omega)$ of the form (66). Using (66) it is easy to integrate (65) to obtain

$$
G(t)=G_{\infty} \delta(t)-\theta(t) \int_{0}^{\infty} \mathrm{d} \tau \frac{H(\tau)}{\tau} \mathrm{e}^{-t / \tau}
$$

or, for a finite set of relaxation times,

$$
G(t)=G_{\infty} \delta(t)-\theta(t) \sum_{k} \frac{H_{k}}{\tau_{k}} \mathrm{e}^{-t / \tau_{k}} .
$$

In appendix B we show in detail how $G(t)$ can be obtained numerically using the procedure described above.

\section{Numerical results: rubber block dynamics}

In this section we present results for the sliding dynamics of a rubber block with thickness $0.5 \mathrm{~cm}$, squeezed against an asphalt road with the nominal pressure $\sigma_{0}=0.4 \mathrm{MPa}$.

Assume first that the upper surface of the rubber block is clamped and undergoes uniform acceleration $a=500 \mathrm{~m} \mathrm{~s}^{-2}$ for $0.004 \mathrm{~s}$. Figure 17 shows (a) the velocities $v_{\mathrm{t}}$ and $v_{\mathrm{b}}$ of the top and bottom surface of the rubber block, respectively, and (b) the shear stress acting on the bottom surface of the rubber block, as a function of the distance $x_{\mathrm{t}}$ the top surface has moved. Note that the bottom surface of the block is effectively pinned until the shear stress reaches $\sigma_{\mathrm{s}} \approx 0.44 \mathrm{MPa}$, corresponding to the friction coefficient $\mu=\sigma_{\mathrm{s}} / \sigma_{0} \approx 1.1$. After the depinning, the velocity of the bottom surface of the block increases towards the velocity of the top surface, while the shear stress approaches a constant value determined by the kinetic friction coefficient. The physical reason for the peak in the shear stress at $x_{\mathrm{t}} \approx 0.07 \mathrm{~cm}$ is due to the flash temperature; the full flash temperature is not built up until the slip distance is of the order of the diameter of the macro-asperity contact regions, i.e. of order $0.4 \mathrm{~cm}$ in the present case.

Next, let us consider a case when the upper surface of the rubber block first accelerates with $a=500 \mathrm{~m} \mathrm{~s}^{-2}$ for $0<t<0.004 \mathrm{~s}$, and then retards with $a=-500 \mathrm{~m} \mathrm{~s}^{-2}$ for 


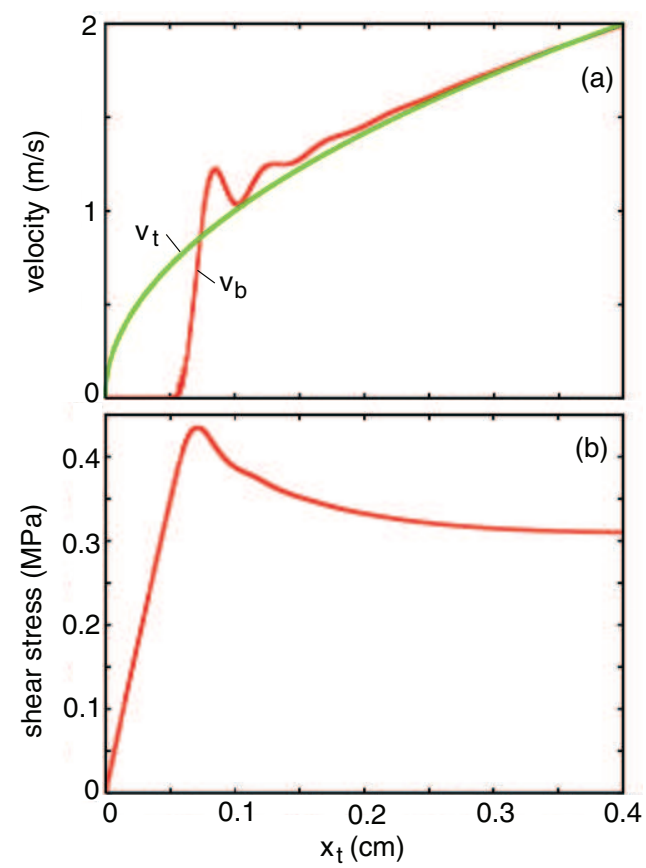

Figure 17. A rubber block with thickness $0.5 \mathrm{~cm}$ sliding on an asphalt road. The nominal pressure $\sigma_{0}=0.4 \mathrm{MPa}$. The upper surface of the rubber block is clamped and undergoes uniform acceleration $a=500 \mathrm{~m} \mathrm{~s}^{-2}$ for $0.004 \mathrm{~s}$. (a) The velocities $v_{\mathrm{t}}$ and $v_{\mathrm{b}}$ of the top and bottom surface of the rubber block, respectively, as a function of the distance $x_{\mathrm{t}}$ the top surface has moved. (b) The shear stress acting on the bottom surface of the rubber block, as a function of $x_{\mathrm{t}}$. In the calculation the block is 'divided' into $N=12$ layers.

$0.004 \mathrm{~s}<t<0.008 \mathrm{~s}$. In figure 18(a) we show the shear stress, divided by the nominal pressure $\sigma_{0}$, acting on the bottom surface of the rubber block, as a function of velocity $v_{\mathrm{t}}$ of the top surface of the block. In figure 18(b) we show the velocity $v_{\mathrm{b}}$ of the bottom surface of the rubber block, as a function of time.

\section{Comparison with experiment}

A lot of experimental data has been presented in the literature related to rubber friction. However, in order to quantitatively compare the rubber friction theory with experimental data, both the viscoelastic modulus $E(\omega, T)$ and the substrate surface roughness power spectrum $C(q)$ must be known. So far this information has only been reported on in two experimental investigations of rubber friction which I am aware of $[9,12,13]$. In the present section we will only consider general (universal) aspects of rubber friction, and show that the theory is in good qualitative agreement with the presented experimental data.

Let us first consider stationary sliding. Figure 19 shows the measured kinetic rubber friction coefficient as a function of the logarithm of the sliding velocity, for a tread rubber block sliding on an asphalt surface [19]. The velocity dependence of $\mu_{\mathrm{k}}(v)$ is in good qualitative agreement with the theory (compare figure 19 with figure 11(a)). More generally, I have found that the theory, and all the experiments known to me, gives a maximal friction coefficient of order unity, and the position of the maximum in the range $10^{-3}-10^{-1} \mathrm{~m} \mathrm{~s}^{-1}$, depending on the rubber compound and the substrate surface. In the absence of the flash temperature, according 

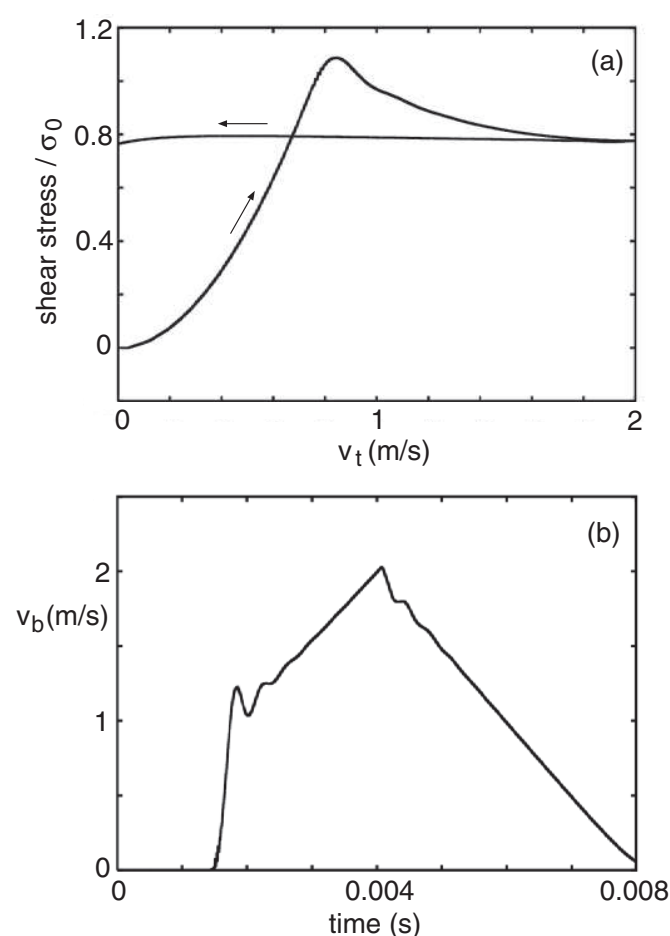

Figure 18. A rubber block with thickness $0.5 \mathrm{~cm}$ sliding on an asphalt road. The nominal pressure $\sigma_{0}=0.4 \mathrm{MPa}$. The upper surface of the rubber block is clamped and undergoes uniform acceleration $a=500 \mathrm{~m} \mathrm{~s}^{-2}$ for $t<0.004 \mathrm{~s}$, and then uniform retardation $a=-500 \mathrm{~m} \mathrm{~s}^{-2}$ for $0.004 \mathrm{~s}<t<0.008 \mathrm{~s}$. (a) The shear stress, divided by the nominal pressure $\sigma_{0}$, acting on the bottom surface of the rubber block, as a function of velocity $v_{\mathrm{t}}$ of the top surface of the block. (b) The velocity $v_{\mathrm{b}}$ of the bottom surface of the rubber block, as a function of time. In the calculation the block is 'divided' into $N=12$ layers.

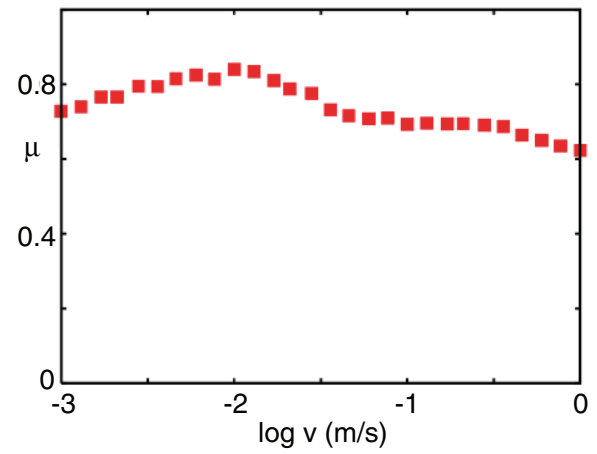

Figure 19. The friction coefficient $\mu$ as a function of the logarithm of the sliding velocity, for a rubber tread block sliding on an asphalt road surface at $T=18^{\circ} \mathrm{C}$. (Courtesy of Olaf Lahayne.)

to the theory the maximum would instead occur at much higher sliding velocities, typically in the range $10^{2}-10^{4} \mathrm{~m} \mathrm{~s}^{-1}$. This illustrates the crucial role of the flash temperature.

Next, let us consider the dependence of the friction coefficient $\mu$ on the background temperature. Figure 20 shows experimental data (obtained using a portable skid tester [17, 18]) 


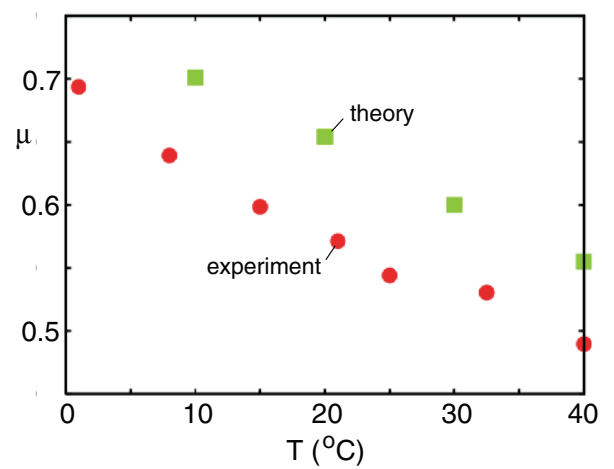

Figure 20. The friction coefficient $\mu$ as a function of the background temperature, during sliding of a rubber block on a rough substrate. The experimental data were obtained using a portable skid tester $[17,18]$ for tread rubber on road surface. The theory data were calculated using the viscoelastic modulus of a tread rubber (blend of several different rubber polymers), sliding on an asphalt road surface (with the power spectrum given in figure 2, surface 1) at $v=1 \mathrm{~m} \mathrm{~s}^{-1}$.

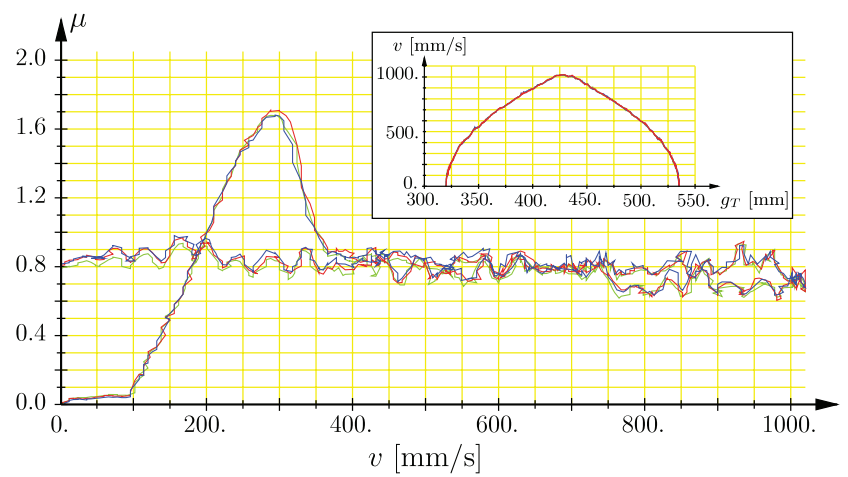

Figure 21. A soft rubber block (thickness $1.1 \mathrm{~cm}$ ) sliding on a wet concrete surface at the nominal pressure $0.2 \mathrm{MPa}$. The variation of the effective friction coefficient with the velocity $v$ of the drive is shown for non-uniform sliding (see inset) involving acceleration $\left(a \approx 5 \mathrm{~m} \mathrm{~s}^{-2}\right)$ followed by retardation $\left(a \approx-5 \mathrm{~m} \mathrm{~s}^{-2}\right.$ ). From reference [20] (with permission).

for a tread rubber block sliding on a road surface. The theory data in the same figure are calculated for a rubber block sliding on an asphalt road surface (with the power spectrum given in figure 2, surface 1) at $v=1 \mathrm{~m} \mathrm{~s}^{-1}$. The viscoelastic modulus of the rubber used in the calculation is for a tread rubber. As expected, the rubber friction decreases with increasing background temperature.

Let us now consider non-stationary sliding. Figure 21 shows results for a (soft) rubber block (thickness $1.1 \mathrm{~cm}$ ) sliding on a wet concrete surface at the nominal pressure $0.2 \mathrm{MPa}$. The variation of the effective friction coefficient with the velocity $v$ of the drive is shown for non-uniform sliding (see inset) involving acceleration $\left(a \approx 5 \mathrm{~m} \mathrm{~s}^{-2}\right)$ followed by retardation $\left(a \approx-5 \mathrm{~m} \mathrm{~s}^{-2}\right)$ [20]. Note that the experimental data are of the general form predicted by the theory (see figure 22) with a 'start-up' peak due to the flash temperature; the rubber block must slide a distance of order the linear size of the macro-asperity contact regions before the full flash temperature has been developed, and this is the origin of the 'start-up' peak.

Note that the 'start-up' peak in figure 21 and in the calculations 22 has nothing to do with the static friction coefficient, but rather is a kinetic effect related to the finite sliding distance 


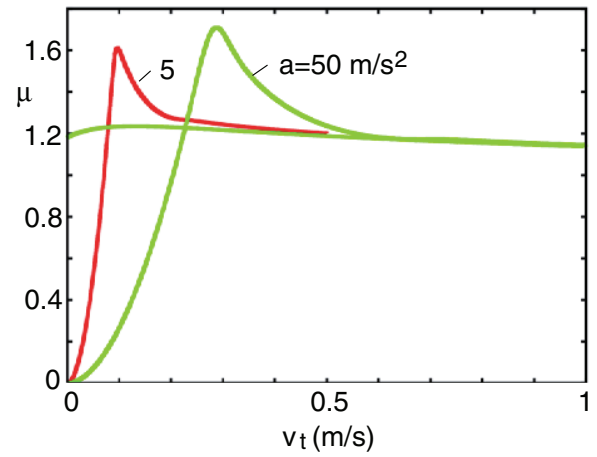

Figure 22. A rubber block (thickness $0.5 \mathrm{~cm}$ ) sliding on an asphalt road surface at the pressure $0.2 \mathrm{MPa}$. The (calculated) variation of the effective friction coefficient with the velocity $v_{\mathrm{t}}$ is shown for two different cases where the drive first accelerates $\left(a \approx 5\right.$ and $50 \mathrm{~m} \mathrm{~s}^{-2}$ ) followed by retardation $\left(a \approx-5\right.$ and $\left.-50 \mathrm{~m} \mathrm{~s}^{-2}\right)$.

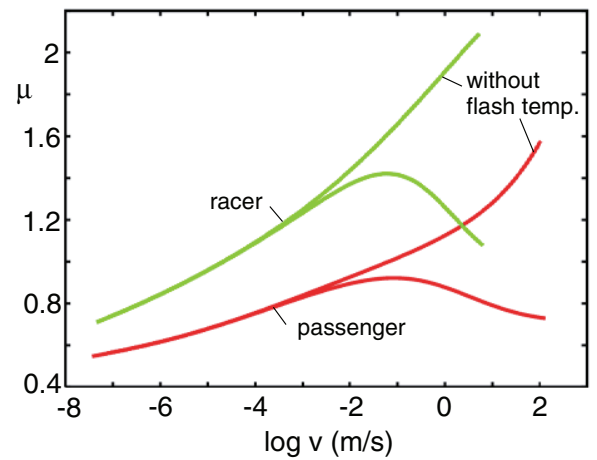

Figure 23. The kinetic (steady state) rubber friction coefficient for typical tyre tread compounds for a racing car (top) and personal car (bottom), sliding on an asphalt road surface. The background temperature $T=60^{\circ} \mathrm{C}$.

necessary in order to fully build up the flash temperature. In fact, rubber on rough substrates does not exhibit any static friction coefficient, but only a kinetic friction which (for small sliding velocities) decreases continuously with decreasing sliding velocity.

Finally, let us check if the theory agrees with the observation that tyres for racing cars (or racing motorcycles) exhibit much larger friction (but also much larger wear) than tyres for passenger cars. In figure 23 I show the calculated kinetic friction coefficient, using the measured viscoelastic modulus of a rubber compound from racing tyres, and for a passenger car tyre, assuming all other conditions identical. The maximum kinetic friction is about $50 \%$ higher for the racing compound, which is also what is typically found experimentally [21]. This calculation indicates that even for racing tyres the main origin of the friction is due to the internal damping of the rubber rather than an adhesive tyre-road interaction.

\section{On the origin of the short distance cut-off}

The rubber friction theory presented above assumes that the friction is due to the internal friction of the rubber. That is, the substrate surface asperities exert forces on the rubber 
surface and result in pulsating deformations of the rubber. The deformations cannot occur completely adiabatically, but result in transfer of energy into random heat motion in the rubber. In calculating this asperity-induced contribution to the friction I include only the road surface roughness with wavevectors $q<q_{1}$. Here I will discuss the origin of the short distance cut-off $q_{1}$. In principle, there may be several different origins of $q_{1}$. For example, if the road is covered by small particles, e.g., dust or sand particles, with typical diameter $D$, then one may expect $q_{1} \approx 1 / D$. Similarly, on a wet road, the water trapped in the surface cavities may act as an effective short distance cut-off [22]. However, for clean dry road surfaces, I believe that the cut-off may be determined by the rubber compound properties.

If no short distance cut-off $q_{1}$ existed the flash temperature and the surface stresses in the contact areas would increase as we study the contact regions at higher and higher magnification. However, when the flash temperature and surface stresses becomes high enough the rubber will degrade [23]. In fact, measurements on bulk samples have shown that natural rubber rapidly thermally degrades already at $T \approx 200^{\circ} \mathrm{C}$. The surface layer of the tyre rubber is exposed to oxygen and ozone and will degrade even faster than in the bulk, even if the temperature is the same. The thermal and stress-induced degradation results in a thin layer of modified rubber at the surface, and we will make the basic assumption that the deformation of the rubber on length scales shorter than the thickness of the modified layer gives a negligible contribution to the tyre-road friction. Thus, with respect to friction, we assume that the modified surface layer acts as a 'dead' layer.

The formation of a modified surface layer will require some run-in time period. This is consistent with the (experimental) information that a freshly prepared rubber surface in general exhibit higher sliding friction than after the run-in time period necessary for the formation of a (fully developed) modified surface layer [24].

The formation of a modified surface layer is a thermally activated mechanical-chemical process involving the breaking of chemical bonds (and the formation of new bonds). The rate of bond breaking (at the temperature $T$ and the tensile stress $\sigma$ ) is assumed to be given by the standard expression of activated processes:

$$
w \approx w_{0} \mathrm{e}^{-\Delta E\left(1-\sigma / \sigma_{\mathrm{c}}\right) / k_{\mathrm{B}} T}
$$

where the attempt frequency $w_{0}$ is usually of order $10^{12} \mathrm{~s}^{-1}$, and $\Delta E$ is the activation energy for bond breaking and $\sigma_{\mathrm{c}}$ the stress necessary for bond breaking at zero temperature. The weakest (chemical) bonds in rubber cross-linked with sulfur are multi-sulfur cross-links, for which $\Delta E$ is of order $\sim 2-3 \mathrm{eV}$. Assuming a typical cross-link density, and that the applied stress distributes itself entirely on the cross-links (which requires such a high temperature that the rubber is liquid-like between the cross-links), and assuming that the same force acts on all the cross-links, one can estimate the stress $\sigma_{\mathrm{c}}$ to be of order $\sim 1 \mathrm{GPa}$. However, in inhomogeneous materials such as rubber, there will be a large distribution of local forces acting on the crosslinks, and the cross-links where the local stress is highest will in general break first.

The contact time of a tyre tread block in the footprint area is typically of order $0.01 \mathrm{~s}$, and since the dead layer has been worn out in about $\sim 100$ contacts the rate $w$ must be of order $1 \mathrm{~s}^{-1}$. Taking $w \sim 1 \mathrm{~s}^{-1}$ gives

$$
\Delta E\left(1-\sigma / \sigma_{\mathrm{c}}\right) / k_{\mathrm{B}} T=\ln \left(w_{0} / w\right) \approx 28 .
$$

Note that the RHS of this expression is not very sensitive to the value of $w$. We can use (70) as a criterion in determining the optimum cut-off $q_{1}$. We determine $q_{1}$ so that equation (70) is satisfied, where $T$ is now the flash temperature in the contact area and $\sigma$ the (shear) stress in the contact area.

We have found that using $\Delta E \approx 1.25 \mathrm{eV}$ and $\sigma_{\mathrm{c}} \approx 100 \mathrm{MPa}$ results in a friction coefficient in good agreement with experiment. Note that $\Delta E$ is smaller than the typical energy to break 
the sulfur cross link (which is of order $2-3 \mathrm{eV}$ ) or the energy to break a $\mathrm{C}-\mathrm{C}$ bond along a carbon chain (which is of order 3-4 eV). However, in the present case the bond breaking is likely to involve reaction with foreign molecules, e.g. ozone or oxygen, and the effective activation energy for such processes may be much smaller than when the bonds break in vacuum. The value for $\sigma_{\mathrm{c}}$ is higher than the rupture stress of macroscopic rubber blocks, but $\sigma_{\mathrm{c}}$ refers to the rupture stress of very small rubber volume elements, which is higher than for macroscopic rubber blocks due to the absence of (large) crack-like defects ${ }^{2}$. It is clear that the processes which determine the cut-off $q_{1}$ are closely related to tyre tread wear.

\section{Summary and conclusion}

When a rubber block slips on a hard rough substrate, the substrate asperities will exert timedependent deformations of the rubber surface resulting in viscoelastic energy dissipation in the rubber, which gives a contribution to the sliding friction. Most surfaces of solids have roughness on many different length scales, and when calculating the friction force it is necessary to include the viscoelastic deformations on all length scales. The energy dissipation will result in local heating of the rubber. Since the viscoelastic properties of rubber-like materials are extremely strongly temperature dependent, it is necessary to include the local temperature increase in the analysis. In this paper I have developed a theory which describes the influence of the flash temperature on rubber friction. At very low sliding velocity the temperature increase is negligible because of heat diffusion, but already for velocities of order $10^{-2} \mathrm{~m} \mathrm{~s}^{-1}$ the local heating may be very important, and I have shown that in a typical case the temperature increase results in a decrease in rubber friction with increasing sliding velocity for $v>0.01 \mathrm{~m} \mathrm{~s}^{-1}$. This may result in stick-slip instabilities, and is of crucial importance in many practical applications, e.g. for tyre-road friction, and in particular for ABS braking systems.

\section{Acknowledgments}

I thank O Albohr for many useful discussions. I also thank Pirelli Pneumatici for support.

\section{Appendix A. Average size of a macro-asperity contact region}

Consider the contact between two solids with nominally flat surfaces. Figure A.1(a)-(d) shows the contact area at increasing magnification. At low magnification $\zeta<1$ it appears as if complete contact occurs between the solids. The macro-asperity contact regions typically appear for a magnification somewhere in the range $\zeta_{\mathrm{m}}=q_{\mathrm{m}} / q_{0} \approx 2-5$ depending on the substrate surface and the rubber compound, where $A / A_{0} \approx 0.25-0.3$ is slightly below the site percolation threshold (for a hexagonal lattice). The macro-asperity contact regions (c) break up into smaller contact regions (d) as the magnification is increased.

Let us now estimate the (average) size of the macro-asperity contact regions. Assume that the contact patches have the surface areas $A_{i}(i=1, \ldots, N)$. Let us define the probability distribution

$$
P_{A}=\frac{1}{N} \sum_{i} \delta\left(A-A_{i}\right)
$$

\footnotetext{
2 A rough estimate of $\sigma_{\mathrm{c}}$ may be obtained from the energy to propagate a crack very slowly in rubber. In this limiting case no viscoelastic energy dissipation takes place in front of the crack tip, and the crack propagation energy (per unit area) (see, e.g., [23]) $G_{0} \approx 2 \pi a_{0} \sigma_{\mathrm{c}}^{2} / E(0)$, giving $\sigma_{\mathrm{c}} \approx\left[G_{0} E(0) / 2 \pi a_{0}\right]^{1 / 2}$, where $E(0)$ is the zero frequency elastic modulus and $a_{0}$ the crack tip radius. In a typical case for styrene butadiene copolymer $G_{0} \approx 30 \mathrm{MPa}$ (see, e.g., [25]) and with $E(0) \approx 2 \mathrm{MPa}$ and $a_{0} \approx 1 \mathrm{~nm}$ we get $\sigma_{\mathrm{c}} \approx 100 \mathrm{MPa}$.
} 

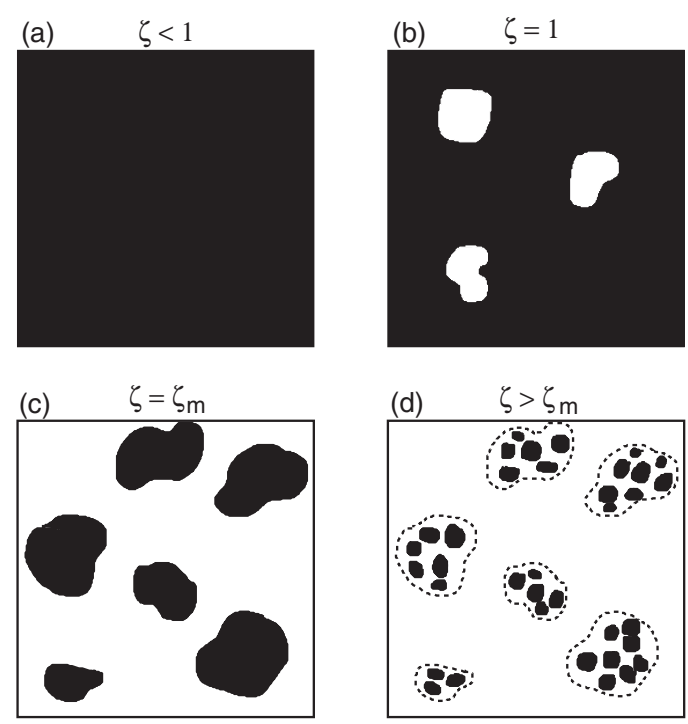

Figure A.1. The contact area at increasing magnification ((a)-(d)). The macro-asperity contact area (c) breaks up into smaller contact areas (d) as the magnification is increased.

where $N$ is the number of contacting asperities. The average area of a contact patch

$$
a=\int \mathrm{d} A P_{A} A=\frac{1}{N} \sum_{i} A_{i} .
$$

Assume that the distribution $P_{h}$ of summit heights is a Gaussian,

$$
P_{h}=\frac{1}{(2 \pi)^{1 / 2} h^{*}} \exp \left[-h^{2} / 2 h^{* 2}\right]
$$

where $h^{*}$ is the root-mean-square amplitude of the summit height fluctuations. Assume that we can neglect the (elastic) interaction between the macro-contact areas. Thus, using the Hertz contact theory the (normalized) area of real contact is [26, 27]

$$
\frac{\Delta A}{A_{0}}=\pi n_{0} R_{0} \int_{d}^{\infty} \mathrm{d} h(h-d) P_{h}
$$

where $A_{0}$ is the nominal contact area, $R_{0}$ the radius of curvature of the asperity, $n_{0}$ the number of asperities per unit area, and $d$ the separation between the surfaces. The number of contacting asperities per unit area

$$
\frac{N}{A_{0}}=n_{0} \int_{d}^{\infty} \mathrm{d} h P_{h} .
$$

Substituting (A.2) in (A.3) and (A.4), and introducing the new integration variable $\xi=$ $(h-d) / h^{*}$, gives

$$
\frac{\Delta A}{A_{0}}=n_{0} R_{0} h^{*}\left(\frac{\pi}{2}\right)^{1 / 2} \int_{0}^{\infty} \mathrm{d} \xi \xi \exp \left[-\frac{1}{2}(x+\xi)^{2}\right]
$$

and

$$
\frac{N}{A_{0}}=\frac{n_{0}}{(2 \pi)^{1 / 2}} \int_{0}^{\infty} \mathrm{d} \xi \exp \left[-\frac{1}{2}(x+\xi)^{2}\right]
$$




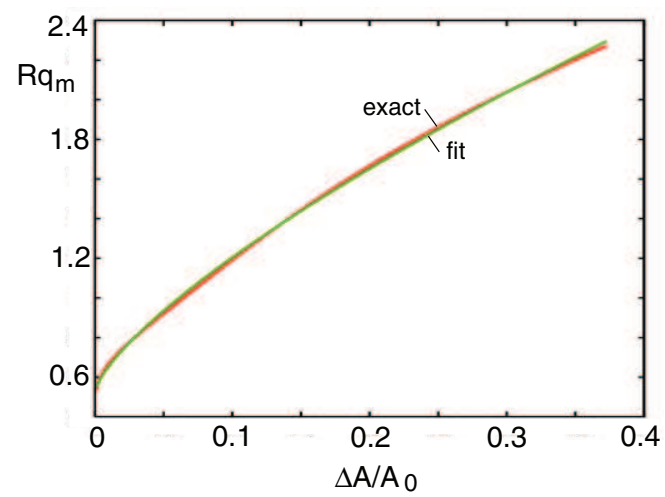

Figure A.2. The (average) radius $R$ of a macro-asperity contact area as a function of the (normalized) projected contact area $\triangle A / A_{0}$.

where $x=d / h^{*}$. Thus, the average macro-asperity contact area

$$
a=\frac{\Delta A}{N}=\frac{\pi R_{0} h^{*} \int_{0}^{\infty} \mathrm{d} \xi \xi \exp \left[-\frac{1}{2}(x+\xi)^{2}\right]}{\int_{0}^{\infty} \mathrm{d} \xi \exp \left[-\frac{1}{2}(x+\xi)^{2}\right]} .
$$

We can estimate the concentration of asperities $n_{0}$, the (average) radius of curvature $R_{0}$ of the asperities, and the rms summit height fluctuation $h^{*}$ as follows. We expect the asperities to form a hexagonal-like (but somewhat disordered) distribution with the lattice constant $\lambda_{\mathrm{m}}=2 \pi / q_{\mathrm{m}}$ so that

$$
n_{0} \approx 2 /\left(\lambda_{\mathrm{m}}^{2} \sqrt{ } 3\right)=\frac{q_{\mathrm{m}}^{2}}{2 \pi^{2} \sqrt{ } 3} \approx 0.029 q_{\mathrm{m}}^{2} .
$$

The height profile along some axis $x$ in the surface plane will oscillate with the wavelength of order $\approx \lambda_{\mathrm{m}}$ roughly as $h(x) \approx h_{1} \cos \left(q_{\mathrm{m}} x\right)$, where $h_{1}=\sqrt{ } 2 h_{0}$ (where $h_{0}$ is the rms roughness amplitude). Thus,

$$
\frac{1}{R_{0}} \approx h^{\prime \prime}(0)=q_{\mathrm{m}}^{2} h_{1}=\sqrt{ } 2 q_{\mathrm{m}}^{2} h_{0}
$$

Finally, we expect the rms of the fluctuation in the summit height to be somewhat smaller than $h_{0}$. In what follows we use [28] $n_{0}=0.023 q_{\mathrm{m}}^{2}, 1 / R_{0}=0.92 h_{0} q_{\mathrm{m}}^{2}$ and $h^{*}=0.63 h_{0}$. Using these results and defining $a=\pi R^{2}$ we obtain from (A.5) and (A.7)

$$
\begin{aligned}
& \frac{\Delta A}{A_{0}}=0.0198 \int_{0}^{\infty} \mathrm{d} \xi \xi \exp \left[-\frac{1}{2}(x+\xi)^{2}\right] \\
& \left(R q_{\mathrm{m}}\right)^{2}=0.688 \frac{\int_{0}^{\infty} \mathrm{d} \xi \xi \exp \left[-\frac{1}{2}(x+\xi)^{2}\right]}{\int_{0}^{\infty} \mathrm{d} \xi \exp \left[-\frac{1}{2}(x+\xi)^{2}\right]} .
\end{aligned}
$$

In figure A. 2 we show the radius $R$ of a macro-asperity contact region as a function of the relative contact area $\Delta A / A_{0}$. The numerical data are well approximated by

$$
R q_{\mathrm{m}}=a+b\left(\Delta A / A_{0}\right)^{c}
$$

where $a=0.526, b=3.636$ and $c=0.729$. This fit function is also shown in figure A.2. In a typical case when a tread block is slipping on a road surface, $A\left(\zeta_{\mathrm{m}}\right) / A_{0}=\Delta A / A_{0} \approx 0.25$. Using figure A. 2 this gives the diameter of the macro-asperity contact regions $2 R \approx 3 / q_{0}$. If $q_{0}=600 \mathrm{~m}^{-1}$ this gives $2 R \approx 0.5 \mathrm{~cm}$. 

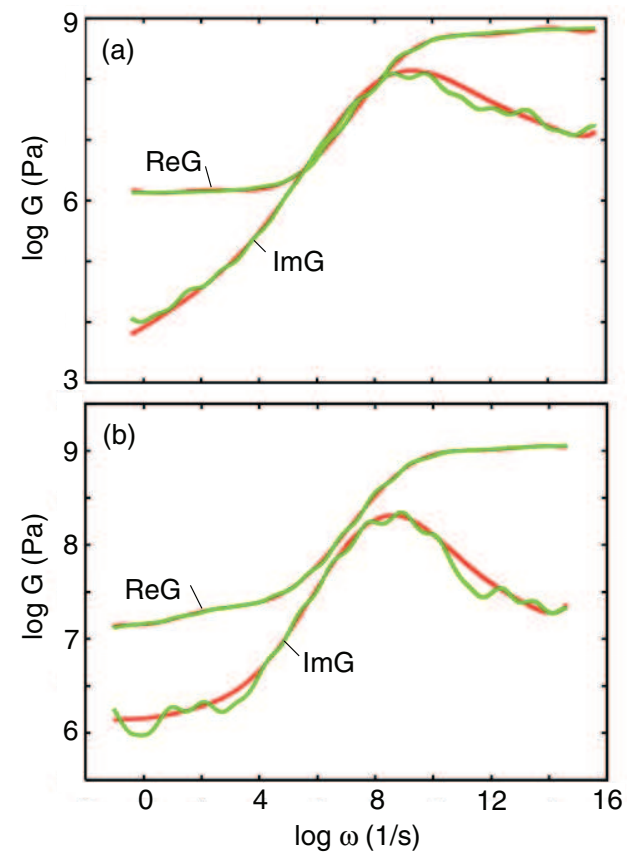

Figure B.1. The real and the imaginary part of the viscoelastic modulus $G(\omega)$ for styrene butadiene copolymer (a) without filler and (b) with $30 \%$ carbon black filler. The red and green lines are the original data and the fit data obtained by fitting the experimental $G(\omega)$ to a sum over relaxation times as described by (68). For the temperature $T=60^{\circ} \mathrm{C}$.

\section{Appendix B}

The minimization of $V$ with respect to $G_{\infty}$ and $H_{k}$ can be performed by using a random number generator: First some (arbitrary) set of parameters $G_{\infty}$ and $H_{k}$ are chosen and $V$ calculated. Next, one replaces $G_{\infty}$ and $H_{k}$ with $G_{\infty}(1+\xi)$ and $H_{k}\left(1+\xi_{k}\right)$, where $\xi$ and $\xi_{k}$ are small random numbers. If the new $V$, calculated using these parameter values, is smaller than the original one, then the new parameters are accepted as improved parameters; otherwise they are rejected and the old parameters kept. If this procedure is repeated (iterated) many times the effective 'potential' $V$ will converge towards a minimum, and the parameters $G_{\infty}$ and $H_{k}$ at the minimum of $V$ are the best possible ones, which we use in calculating $G(t)$ as described in section 7.

As an example, in figure B.1(a) we show the real and the imaginary part of the viscoelastic modulus $G(\omega)$ for styrene butadiene copolymer (a) without filler and (b) with $30 \%$ carbon black filler [9]. The measuring procedure for obtaining $G(\omega)$ is described in detail [9], and here we only point out that the measurements were performed at $0.5 \%$ strain amplitude. The red and green lines are the experimental data and the fit data, respectively, where the fit data were obtained by approximating the experimental $G(\omega)$ by the sum (68) as described above. Note that for the unfilled rubber both the real and the imaginary part of $G(\omega)$ are very well fitted by suitable choice of a single real (positive) function $H(\tau)$. This is possible only because $\operatorname{Re} G(\omega)$ and $\operatorname{Im} G(\omega)$ are not independent but related via a Kramers-Kronig relation (see below). For the filled rubber the fit of $\operatorname{Re} G$ is equally good, but there is a small deviation for $\operatorname{Im} G$. We have observed the same for other rubber compounds, and sometimes the deviation $\Delta G$ between measured and fitted $\operatorname{Im} G$ is larger than in figure B.1(b), but the calculated $G(t)$ 


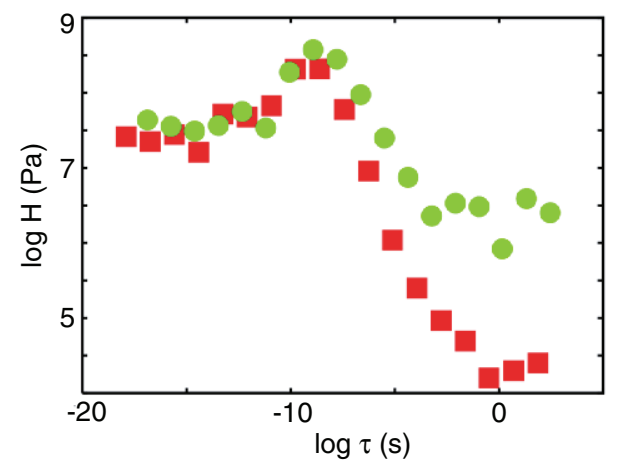

Figure B.2. The spectral density $H_{k}$ as a function of the relaxation time $\tau_{k}$ as obtained by fitting the measured $G(\omega)$ to the spectral representation (68). The squares and circles correspond to the unfilled and filled SB copolymer. For the temperature $T=60^{\circ} \mathrm{C}$.

is still accurate enough for our applications. The deviation $\Delta G$ reflects the fact that for filled rubber $G(\omega)$ is not a 'perfect' linear response function, but exhibits non-linearity. One source of non-linearity is the so-called Payne effect, due to the strain-induced break-up of the filler network $[15,16]$.

Figure B. 2 shows the spectral density $H_{k}$ as a function of the relaxation time $\tau_{k}$ as obtained by fitting the measured $G(\omega)$ to the spectral representation (68). The squares and circles correspond to the unfilled and filled SB copolymers. Note that the main difference between the two cases occurs for the longest relaxation times $\tau>10^{-8} \mathrm{~s}$, where the filled rubber has a much higher spectral density $H(\tau)$. This implies that for the filled rubber, in contrast to unfilled rubber, there is a high spatial density of (relatively) high energy barrier rearrangement processes. This results from polymer molecules bound to the surfaces of the carbon filler particles; the bound layers, which may be $\sim 1 \mathrm{~nm}$ thick, are in a glassy-like state and exhibit larger energy barriers for (thermally activated) rearrangements, as compared to the polymer segments further away from the filler particles.

We emphasize that only when $G(\omega)$ corresponds to a linear response function will causality result in a well defined analytical structure for $G(\omega)$. In this case the real part $G_{1}=\operatorname{Re} G$ and imaginary part $G_{2}=\operatorname{Im} G$ of $G(\omega)$ are related via Kramers-Kronig relations:

$$
\begin{aligned}
& G_{1}(\omega)-G_{\infty}=\frac{1}{\pi} P \int_{-\infty}^{\infty} \mathrm{d} \omega^{\prime} \frac{G_{2}\left(\omega^{\prime}\right)}{\omega^{\prime}-\omega}, \\
& G_{2}(\omega)=-\frac{1}{\pi} P \int_{-\infty}^{\infty} \mathrm{d} \omega^{\prime} \frac{G_{1}\left(\omega^{\prime}\right)-G_{\infty}}{\omega^{\prime}-\omega},
\end{aligned}
$$

where $G_{\infty}=G(\infty)$ is the (real) high frequency shear modulus. For filled rubbers, (B.1) and (B.2) only hold approximately. In particular, due to the Payne effect (see above), the rubber behaves in a non-linear way, i.e. $G(\omega)$ is not a 'perfect' linear response function, and equations (B.1) and (B.2) will only hold approximately.

\section{References}

[1] Moore D F 1972 The Friction and Lubrication of Elastomers (Oxford: Pergamon)

[2] Persson B N J 1998 Surf. Sci. 401445

[3] Persson B N J 2002 Phys. Rev. Lett. 89245502

[4] Galliano A, Bistac S and Schultz J 2003 J. Colloid Interface Sci. 265372 
Galliano A, Bistac S and Schultz J 2003 J. Adhes. 79973

[5] Persson B N J 2002 Eur. Phys. J. E 8385

[6] Grosch K A 1974 The Physics of Tire Traction: Theory and Experiment ed D F Hays and A L Browne (New York: Plenum) p 143

[7] Persson B N J and Volokitin A I 2002 Phys. Rev. B 65134106

[8] Klüppel M and Heinrich G 2000 Rubber Chem. Technol. 73578

[9] Le Gal A and Klüppel M 2005 J. Chem. Phys. 123014704

[10] Persson B N J 2001 J. Chem. Phys. 1153840

[11] Yang C, Tartaglino U and Persson B N J 2006 Eur. Phys. J. E 1947

[12] Westermann S, Petry F, Boes R and Thielen G 2004 Tire Technology International_Annual Review 2004 p 68 (Edited by UKIP Media \& Events, UK)

[13] Westermann S, Petry F, Boes R and Thielen G 2004 Kautschuk Gummi Kunststoffe 57645

[14] Williams M L, Landel R F and Ferry J D 1955 J. Am. Chem. Soc. 773701

[15] Payne A R 1972 J. Appl. Polym. Sci. 161191

[16] Heinrich G and Klüppel M 2002 Adv. Polym. Sci. 1601

[17] Ohhara R 1996 Int. Polym. Sci. Technol. 2325

[18] Sakai Y 1967 Jidosha Gijutsu 21640

[19] Lahayne O 2006 private communication

[20] Huemer T, Liu W N, Eberhardsteiner J, Mang H A and Meschke G 2001 KGK Kautschuk Gummi Kunststoffe 54 458

[21] Haney P 2003 The Racing \& High-Performance Tire Co-published by TV MOTERSPORTS and SAE

[22] Persson B N J, Tartaglino U, Albohr O and Tosatti E 2004 Nat. Mater. 3882

[23] Persson B N J, Albohr O, Heinrich G and Ueba H 2005 J. Phys.: Condens. Matter 17 R1071

[24] Molten J 2003 Fortbildungsseminar Elastomerreibung und Kontactmechanik (Hannover: Deutsches Institute für Kautschuktechnologie eV)

[25] Gent A N 1996 Langmuir 124492

[26] Greenwood J A 1992 Fundamentals of Friction, Macroscopic and Microscopic Processes ed I L Singer and H M Pollack (Dordrecht: Kluwer)

[27] Greenwood J A and Williamson J B P 1966 Proc. R. Soc. A 295300

[28] Nayak P R 1971 ASME J. Lubrication Technol. 93398 